\title{
ON PARABOLIC AND UMBILIC POINTS OF IMMERSED HYPERSURFACES
}

\author{
BY \\ E. A. FELDMAN( ${ }^{(1)}$
}

Introduction. This paper is a natural sequel to Geometry of immersions. I and II ([1] and [2]). The object is to apply the general geometric singularity theory developed in [1] and [2] to two specific geometric singularities of an immersed $n$ manifold in $R^{n+1}$. These applications differ from those at the end of [2] as they involve a more detailed study of the singularity in question which sharpens the information given by the general theory. In $\$ 1$ we review much of the necessary information from [1] and [2]. In $\$ 2$ we set up the machinery for studying the parabolic points of an immersion of a compact $n$-manifold into $R^{n+1}$ as a type of singularity of the osculating map (a refinement of the 2-jet). We also apply the main theory in $\$ 2$. $\S 3$ refines the results of $\S 2$, especially in the case $n=2$. In $\S 4$ and $\S 5$ we repeat the same process for umbilic points. We will close by illustrating how we proceed in the case of the umbilic points.

Let $X$ be a compact 2-dimensional manifold. Let $I\left(X, R^{3}\right)$ be the set of immersions of $X$ in $R^{3}$. Let $f \in I\left(X, R^{3}\right)$. We ask when if ever is the set of umbilics (points where the two principal curvatures are equal) a submanifold of codimension two, and if it is a submanifold in a particularly "nice" way, we ask how many umbilics there are. An application of the two main theorems [1] and [2] (and §1) gives the following answer. For an open dense subset $D_{U}$ of $I\left(X, R^{3}\right)$, the number of umbilic points is even. This is roughly the content of $\S 4$. In $\S 5$ the transversality condition of "niceness" is studied in detail and is related to the index of an isolated umbilic point. The main result is: if $f \in D_{U}$ then the index of an umbilic point of $f$ is $\pm \frac{1}{2}$, and therefore the number of umbilic points of $f$ is greater than or equal to $2|\chi(X)|$ where $\chi(X)$ is the Euler characteristic.

A more complete outline of these ideas is found in the last sections of [3]. The material in $\$ 2$ and $\$ 4$ was contained in the author's doctoral dissertation at Columbia University. $\S 3$ and $\S 5$ are new. Finally, in addition to the people thanked in [1] and [2], I would like to thank J. Milnor for suggesting that 5.6 might be true.

0. Notations and conventions. (a) All manifolds discussed in this paper will be finite dimensional and all the connected components of a given manifold will have the same dimension. The manifolds will satisfy the second axiom of countability

Received by the editors February 8, 1965 and, in revised form, September 13, 1966.

(1) This research was supported by the following contracts: NONR-266(57) and NSF 19022 at Columbia University and NSF GP-2425 at Princeton University. Part of this paper is contained in the author's doctoral dissertation at Columbia University. 
and will be of class $C^{\infty}$. We will use the adjectives smooth or differentiable to connote a manifold or a map between manifolds of class $C^{\infty}$.

(b) If $X$ and $Y$ are smooth manifolds, we will denote by $C^{k}(X, Y)$ the set of smooth maps from $X$ to $Y$ with the "fine" $C^{k}$ topology, and $C(X, Y)$ the set of smooth maps with the fine $C^{\infty}$ topology. This topology is merely the union of all the open sets of the $C^{k}$ topologies. It is a topology because it coincides with the inverse limit topology on $C(X, Y)$ viewing the $C^{k}(X, Y)$ with the obvious inclusion maps as an inverse mapping system. For further details on this topology we refer the reader to the appendix at the end of [1]. All the theorems in this paper are stated in their " $C^{\infty}$ " form; it is clear that they are also true " $C^{k}$ " for $k$ sufficiently large. The $k$ can be determined by counting the number of derivatives used and comparing that number with the restrictions imposed by the Sard theorem. Finally let $I(X, Y)$ denote the set of smooth immersions of $X$ in $Y$. It is well known that $I(X, Y)$ is open in $C(X, Y)$.

(c) If $\xi=(\pi: E \rightarrow X)$ is a smooth vector bundle we will denote by $S(E)$ (sometimes $S(\xi)$ ) the space of smooth cross sections of $\xi$ topologized as a subset of $C(X, E)$. We will denote by $F(X)$ the space of smooth real valued functions $C(X, R)$.

(d) Let $\xi=(\pi: E \rightarrow X)$ and $\eta=\left(\pi^{\prime}: E^{\prime} \rightarrow Y\right)$ be smooth vector bundles. Denote by $\operatorname{Hom}(\xi, \eta)$ (or by an abuse of notation $\operatorname{Hom}\left(E, E^{\prime}\right)$ ) the vector bundle over $X \times Y$ whose fiber over the point $(x, y) \in X \times Y$ is the vector space $\operatorname{Hom}\left(E_{x}, E_{y}^{\prime}\right)$. This set is made into a vector bundle in the obvious way. Let $D: X \rightarrow X \times X$ be the diagonal map, and let $\xi=(\pi: E \rightarrow X)$ and $\eta=\left(\pi^{\prime}: E^{\prime} \rightarrow X\right)$ be smooth vector bundles. We shall denote by Hom $\left(E, E^{\prime}\right) \mid X$ the pull back bundle $D^{-1}\left(\operatorname{Hom}\left(E, E^{\prime}\right)\right)$ over $X$. This latter bundle (the one we denoted by $\left.\operatorname{Hom}\left(E, E^{\prime}\right) \mid X\right)$ is the bundle more usually denoted by $\operatorname{Hom}\left(E, E^{\prime}\right)$.

(e) Let $\xi=(\pi: E \rightarrow X)$ be a smooth vector bundle. Then we denote by $O^{2} E$ the twofold symmetric tensor product of $E$ with itself. Let $\eta=\left(\pi^{\prime}: E^{\prime} \rightarrow Y\right)$ be another vector bundle, we connote by the expression " $g: E \rightarrow E^{\prime}$ is a vector bundle homomorphism covering $f: X \rightarrow Y$ " the commutative diagram of smooth maps on the left, where $g$ maps the fiber $E_{x}$ linearly into the fiber $E_{f(x)}^{\prime}$.

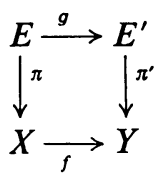

(f) The following number appears very frequently, so we have set aside a special notation for it. Let

$$
\nu(n)=n+n(n+1) / 2 .
$$

(g) We recall that a submanifold collection $K=K_{1} \cup \cdots \cup K_{s}$ of submanifolds of a manifold $X$ is a finite union of disjoint regular submanifolds $K_{i}$, such that $\operatorname{dim} K_{i}>\operatorname{dim} K_{i+1}$, and for each $i, K_{i} \cup K_{i+1} \cup \cdots \cup K_{s}$ is closed in $X$. 
(h) Let $X, Y$, and $N$ be three smooth manifolds. Assume $N$ is a submanifold of $Y$ and let $f: X \rightarrow Y$ be a smooth map. We write $f(X) \hbar N$ to mean $f$ is transversal to $N$ in $Y$.

1. A review. In this section we will review the necessary material contained in [1], [2], and [4].

Let $X$ be a smooth manifold. Let $T_{1}(X)$ denote the tangent bundle of $X$ and let $T_{2}(X)$ denote the second order tangent bundle of $X$. If $x \in X$, and if $\left(x_{1}, \ldots, x_{n}\right)$ are coordinate functions in a coordinate neighborhood containing $x$, then $T_{2}(X)_{x}$ is the subspace of $\operatorname{Hom}_{R}(F(X), R)$ containing functionals of the form

$$
\left.\sum_{1 \leqq i \leqq n} a_{i} \frac{\partial}{\partial x_{i}}\right|_{x}+\left.\sum_{1 \leqq i \leqq j \leqq n} a_{i j} \frac{\partial^{2}}{\partial x_{i} \partial x_{j}}\right|_{x}
$$

$T_{2}(X)$ is made into a vector bundle by using the chain rule. It is easy to see that there is a canonical injection of $T_{1}(X)$ into $T_{2}(X)$ which we will denote by $I$, and that we have the following short exact sequence of vector bundles over $X$,

$$
0 \longrightarrow T_{1}(X) \stackrel{I}{\longrightarrow} T_{2}(X) \stackrel{P}{\longrightarrow} T_{1}(X) 0 T_{1}(X) \longrightarrow 0 .
$$

Let $Y$ be another smooth manifold, and let $f: X \rightarrow Y$. We can then define a smooth vector bundle homomorphism $T_{2}(f): T_{2}(X) \rightarrow T_{2}(Y)$ covering $f: X \rightarrow Y$. Let $x \in X$ and $y=f(x) \in Y$. Let $U$ and $V$ be respectively coordinate neighborhoods of $x$ and $y$ such that $f(U) \subseteq V$. Let $\left(x_{1} \cdots x_{n}\right)$ and $\left(y_{1} \cdots y_{N}\right)$ be respectively coordinate functions on $U$ and $V$, and set $f_{i}\left(x_{1} \cdots x_{n}\right)=y_{i} f\left(x_{1}, \ldots, x_{n}\right)$. We then know that

$$
\left.T_{2}(f) \frac{\partial}{\partial x_{i}}\right|_{x}=\left.\sum_{1 \leqq j \leqq N} \frac{\partial f_{j}}{\partial x_{i}} \frac{\partial}{\partial y_{j}}\right|_{y},
$$

and

$$
\begin{aligned}
T_{2}(f) \frac{\partial^{2}}{\partial x_{i} \partial x_{j}}= & \left.\sum_{1 \leqq k \leqq N} \frac{\partial^{2} f_{k}}{\partial x_{i} \partial x_{j}} \frac{\partial}{\partial y_{k}}\right|_{y}+\left.\sum_{1 \leqq k \leqq N} \frac{\partial f_{k}}{\partial x_{i}} \frac{\partial f_{k}}{\partial x_{j}} \frac{\partial^{2}}{\partial y_{k}^{2}}\right|_{y} \\
& +\left.\sum_{1 \leqq k<e \leqq N}\left(\frac{\partial f_{k}}{\partial x_{i}} \frac{\partial f_{e}}{\partial x_{j}}+\frac{\partial f_{k}}{\partial x_{j}} \frac{\partial f_{e}}{\partial x_{i}}\right) \frac{\partial^{2}}{\partial y_{k} \partial y_{e}}\right|_{y}
\end{aligned}
$$

$T_{2}(f)$ is defined by the manner in which $f^{*}: F(Y) \rightarrow F(X)$ in the same way that we define $T_{1}(f)$. Finally we have the following commutative diagram with exact rows,

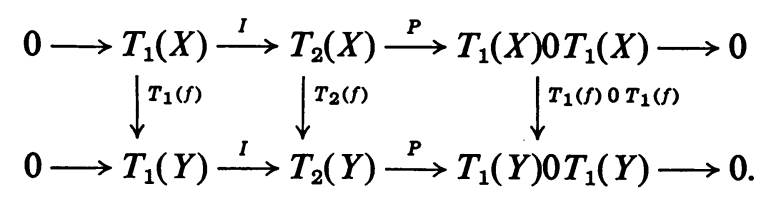

For the proofs of these facts and more details, see $§ 1$ of [1] and Chapter II of [4]. 
Let $Y=R^{N}$. Let us choose an inner product on $Y$, and an orthonormal basis (coordinates), $\left(y_{1} \cdots y_{N}\right)$. Our choice of Euclidean structure defines a map $w_{2}: T_{2}(Y) \rightarrow T_{1}(Y)$ which splits the sequence

$$
0 \rightarrow T_{1}(Y) \rightarrow T_{2}(Y) \rightarrow T_{1}(Y) 0 T_{1}(Y) \rightarrow 0 .
$$

In our global coordinates

$$
w_{2}\left(\left.\left(\partial / \partial y_{i}\right)\right|_{y}\right)=\left.\left(\partial / \partial y_{i}\right)\right|_{y} \text { and } w_{2}\left(\left.\left(\partial^{2} / \partial y_{i} \partial y_{j}\right)\right|_{y}\right)=0
$$

for every $y \in Y$. In general if $Z$ is an arbitrary manifold one can show that splittings of $0 \rightarrow T_{1}(Z) \rightarrow T_{2}(Z) \rightarrow T_{1}(Z) 0 T_{1}(Z) \rightarrow 0$ are in one-one correspondence with symmetric linear connections on $Z$ (see $[1, \S 2])$. From now on when we speak of $R^{N}$ we will assume that we have fixed a Euclidean structure on it, and hence have determined a splitting $w_{2}: T_{2}\left(R^{N}\right) \rightarrow T_{1}\left(R^{N}\right)$.

Definition 1.1. Let $X$ be a smooth manifold, and let $f \in C\left(X, R^{N}\right)$. Let us set $O(f)=O_{2}(f)=w_{2} \circ T_{2}(f): T_{2}(X) \rightarrow T_{1}\left(R^{N}\right) . \quad O_{2}(f)$ is a vector bundle homomorphism covering $f: X \rightarrow R^{N}$. $O_{2}(f)$ is called the osculating map associated to $f$. (Note that $\mathrm{O}_{2}$ depends upon the Euclidean structure in $R^{N}$ that we chose at the outset. See $[1, \S 3]$.) $O(f)$ induces a map $O(f)^{\wedge}: X \rightarrow \operatorname{Hom}\left(T_{2}(X), T_{1}\left(R^{N}\right)\right)$, which is a cross section over the graph of $f$, and is defined by $O(f)^{\wedge}(x)=O(f)_{x} \mid T_{2}(X)_{x}$, for all $x \in X$.

Let us assume $X$ is an $n$-dimensional manifold. It is easy to see $([1, \S 1])$ that $T_{2}(X)$ is a vector bundle that is naturally associated to a principal $J^{2}(n)$ bundle over $X$, where $J^{2}(n)$ is the group of invertible two jets from $R^{n}$ to $R^{n}$ with source and target the origin. Hence we can consider Hom $\left(T_{2}(X), T_{1}\left(R^{N}\right)\right)$ as a bundle with structural group $J^{2}(n) \times O(N)$. The fiber dimension of $T_{2}(X)$ is $\nu(n)=\nu=n+n(n+1) / 2$, hence the fiber of Hom $\left(T_{2}(X), T_{1}\left(R^{N}\right)\right)$ is just Hom $\left(R^{v(n)}, R^{N}\right)$.

DEFINITION 1.2. Let $K=K_{1} \cup \cdots \cup K_{s}$ be a submanifold collection of

$$
\operatorname{Hom}\left(R^{v(n)}, R^{N}\right)=F
$$

which is invariant under the action of $J^{2}(n) \times O(N)$ on $F . K$ is called a model second order Euclidean singularity (or just a model singularity if no confusion can arise). $K$ defines in the obvious way a submanifold collection

$$
K\left(X, R^{N}\right)=K_{1}\left(X, R^{N}\right) \cup \cdots \cup K_{s}\left(X, R^{N}\right)
$$

of Hom $\left(T_{2}(X), T_{1}\left(R^{N}\right)\right)$, where codim $K_{i}\left(X, R^{N}\right)=\operatorname{codim} K_{i} . K\left(X, R^{N}\right)$ is called the Euclidean singularity induced by $K$. Let $f \in C\left(X, R^{N}\right)$. If $O(f)^{\wedge}(x) \in K\left(X, R^{N}\right), x$ is called a $K$-singular point of $O(f)$ or just a $K$-singular point of $f$ if no confusion can arise. (See [2, $\$ \$ 1$ and 3] for more details.)

The following is a corollary of our main transversality theorem (see $\$ 4$ of [1], and $\S 3$ of [2]). 
THEOREM 1.3. (a) Let $X$ be a smooth manifold of dimension $n$, and let

$$
K \subseteq \operatorname{Hom}\left(R^{v(n)}, R^{N}\right)
$$

be a second order Euclidean singularity. Let $K\left(X, R^{N}\right)$ be the induced singularity in Hom $\left(T_{2}(X), T_{1}\left(R^{N}\right)\right)$. Then the set of $f \in C\left(X, R^{N}\right)$, such that $O(f)^{\wedge}(X)$ meets $K\left(X, R^{N}\right)$ transversally, is dense in $C\left(X, R^{N}\right)$.

(b) If $K=K_{1} \cup \cdots \cup K_{s}$, and if the codimension of $K_{2}$ is greater than $n$, then the set of $f \in C\left(X, R^{N}\right)$, such that $O(f)^{\wedge}(X)$ meets $K\left(X, R^{N}\right)$ transversally, is open in $C\left(X, R^{N}\right)$.

The following is a corollary of the main polynomial theorem. (See Theorem 2.5 of [2].)

THEOREM 1.4. Let $X$ be a smooth connected n-dimensional manifold. Let

$$
K \subseteq \operatorname{Hom}\left(R^{v(n)}, R^{N}\right)
$$

be a Euclidean singularity and let us assume that $K$ is a real algebraic variety. Let $K\left(X, R^{N}\right) \subseteq \operatorname{Hom}\left(T_{2}(X), T_{1}\left(R^{N}\right)\right)$ be the singularity induced by $K$. Furthermore assume that $O(f)^{\wedge}(X)$ meets $K\left(X, R^{N}\right)$ transversally.

(a) Then $K(f)=\left(O(f)^{\wedge}\right)^{-1}\left(K\left(X, R^{N}\right)\right)$, the set of $K$ singular points of $f$ is a submanifold collection of $X$ of the same codimension as $K \subseteq \operatorname{Hom}\left(R^{v}, R^{N}\right)$.

(b) Furthermore $K(f)$ carries a fundamental homology class $[K(f)]\left(Z_{2}\right.$ coefficients of course).

(c) There exists a universally defined polynomial $\boldsymbol{P}$ which is independent of $f$, and in fact depends only on $K$, such that $D_{X} i_{*}[K(f)]=\boldsymbol{P}\left(W_{i}(X)\right)$, where $i: K(f) \rightarrow X$ is the inclusion map, $D_{X}$ is Poincaré duality in $X$, and $W_{i}(X)$ are the Stiefel-Whitney classes of $X$.

Let $X$ be a connected $n$-dimensional manifold, such that $I\left(X, R^{n+1}\right)$ is nonempty. Let $f \in I\left(X, R^{n+1}\right)$ and let $N_{f}(X)$ be the normal bundle of $f$. We then have the following commutative diagram with homomorphisms over $X$ of vector bundles and vector bundle exact rows and columns.

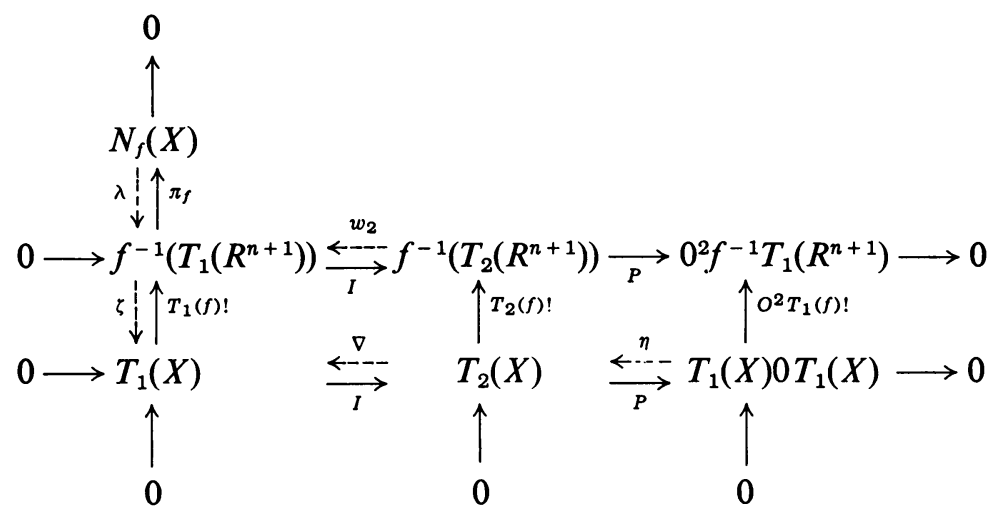

Diagram 1.1 
The bundles on the second row are the pull backs by $f$ of the original exact sequence of bundles over $R^{n+1}$. The vertical maps from the first row to the second are the vector bundle homomorphism over $X$ factor of the canonical factoring of a vector bundle homomorphism into a bundle map over $f$ and a vector bundle homomorphism over $X . w_{2}$ is the pull back of the splitting of the second row over $R^{n+1}, \pi_{f}$ is the canonical projection onto the cokernel, $\zeta$ is the splitting of the vertical sequence induced by the (Riemann) Euclidean structure on $R^{n+1}, \lambda$ the splitting "on the top" induced by $\zeta, \nabla=\zeta w_{2} T_{2}(f)$ ! the induced Riemann connection on $X$ (see $[1, \S \S 2,9]$ and $[2, \S 5]$ ) is a splitting of the first row, and $\eta$ is the induced splitting "on the right."

DEFINITION 1.5. (a) Let $\nu_{f}=\pi_{f} w_{2} T_{2}(f) ! \eta . \quad \nu_{f}: T_{1}(X) 0 T_{1}(X) \rightarrow N_{f}(X)$ is called the (second) normal form of $f$.

(b) Let $N \in S\left(N_{f}(X)\right)$, and let $\langle$, $\rangle$ be the Riemann structure $f$ induces on $T_{1}(X)$ and $\langle,\rangle^{1}$ the Riemann structure it induces on $N_{f}(X) . N$ induces a vector bundle homomorphism (over $X$ ) $S_{N}: T_{1}(X) \rightarrow T_{1}(X)$ which is defined by

$$
\left\langle S_{N}(x) v, w\right\rangle_{x}=\left\langle\nu_{f}(v, w), N(x)\right\rangle_{x}^{1} \quad \text { for all } x \in X
$$

and $u, v \in T_{1}(X)_{x} . S_{N}$ is clearly self adjoint (for each $x \in X$ ) and is called the operator of the second fundamental form of the immersion $f$.

(c) Let $N$ be as in (b). For each $x \in X$ we can then define a quadratic form $Q_{N}(x)$ on $T_{1}(X)_{x}$ by the formula

$$
Q_{N}(x)(u, v)=\left\langle S_{N}(x) v, u\right\rangle_{x} \quad \text { for } u, v \in T_{1}(X)_{x} .
$$

$Q_{N}$ is called the second fundamental form of $f$.

Definition 1.6. Let $f \in I\left(X, R^{n+1}\right)$ and let $\nu_{f}: T_{1}(X) 0 T_{1}(X) \rightarrow N_{f}(X)$ be the normal form of $f$. We say $x \in X$ is a totally geodesic point if $v_{f} \mid T_{1}(X)_{x} 0 T_{1}(X)_{x}$ is identically zero.

More information about $\nu_{f}, S_{N}$, and $Q_{N}$ can be found in $\S 5$ of [2], and a fairly complete study of totally geodesic points appears in $\$ 7$ of [2].

2. Parabolic points. I. Before entering the main text of this section, we will set up some notations and conventions which will be useful in this and the following sections. Let $\left(y_{1}, \ldots, y_{N}\right)$ be the usual global coordinates for $R^{N}$ gotten by considering $R^{N}$ as $N$-tuples of real numbers. Hence at the origin of $R^{N}$ we have the unit vectors $e_{i}=(0, \ldots, 0,1,0, \ldots, 0)$ with the 1 in the $i$ th place. Endow $R^{N}$ with its usual Riemann structure. As is well known $T\left(R^{N}\right) \cong R^{N} \times R^{N}$. Hence each tangent vector $v$ to $R^{N}$ at $p$ will be denoted by a $2 N$-tuple of reals $\left(p_{1}, \ldots, p_{N} ; v_{1}, \ldots, v_{N}\right)$. We clearly have the $N$ orthonormal vector fields $\tilde{e}_{1}, \ldots, \tilde{e}_{N}$ on $R^{N}$ given by $\tilde{e}_{i}(p)=\left(p, e_{i}\right)$. Furthermore for each $p \in R^{N}$ we have a map $A_{p}: T\left(R^{N}\right)_{p} \rightarrow R^{N}$ given by $A_{p}\left(p ; v_{1}, \ldots, v_{N}\right)=\left(v_{1}, \ldots, v_{N}\right) . A_{p}$ is an isometry for each $p$ and can be considered as the projection onto the fiber. We will often use $A_{p}$ to identify $T\left(R^{N}\right)_{p}$ with $R^{N}$. Let $w_{2}: T_{2}\left(R^{N}\right) \rightarrow T\left(R^{N}\right)$ be the splitting given by the above Riemann structure. Furthermore, we will adopt the notation of Diagram 1.1 of 
the last section. Finally we will carry the following two assumptions throughout this and the next sections. Let $X$ be a smooth $n$-dimensional manifold with $n \geqq 2$. The most crucial assumption is that the set $I\left(X, R^{n+1}\right)$ of smooth immersions of $X$ in $R^{n+1}$ is nonempty. Let $T_{2}(X) \rightarrow T_{1}\left(R^{n+1}\right)$ be the osculating map $O(f)$.

Definition 2.1. Let $f \in I\left(X, R^{n+1}\right)$. Let $\xi: f^{-1} T_{1}\left(R^{n+1}\right) \rightarrow T_{1}(X)$ be the splitting of the vertical exact sequence of Diagram 1.1 induced by the Riemann structure on $R^{n+1}$. Let $N_{f}(X)$ and $\nu_{f}$ be the normal bundle and the normal form of the immersion $f$ respectively. Let $x \in X$. Let $U$ be a suitably small neighborhood of $x$. Let $N \in S\left(N_{f}(X)\right)$, such that $\|\lambda N(y)\|_{y}=1$ for every $y \in U$. Let $S_{N}$ be the operator of the second fundamental form, of the immersion $f$.

Then $S_{N}(x): T(X)_{x} \rightarrow T(X)_{x}$ linearly. Then set

$$
K_{N, f}(x)=\operatorname{det}\left(S_{N}(x)\right) \text {. }
$$

$K_{N, f}(x)$ is called the Gauss-Kronecker curvature of $f$ at $x$, with respect to the normal direction $N$.

We then set

$$
K_{f}(x)=\frac{1}{2}\left(\operatorname{det}\left(S_{N}(x)\right)+\operatorname{det}\left(S_{-N}(x)\right)\right) .
$$

$K_{f}$ is called the mean Gauss-Kronecker curvature of $f$ at $x$.

In that $S_{N}(x)$ is a self adjoint operator on $T(X)_{x}$, we know that

$$
\operatorname{det}\left(S_{N}(x)\right)=(-1)^{n} \operatorname{det}\left(S_{-N}(x)\right) \text {. }
$$

Hence

$$
\begin{aligned}
K_{f}(x) & =0 & & \text { if } n \text { is odd, } \\
& =K_{N, f}(x) & & \text { if } n \text { is even. }
\end{aligned}
$$

We furthermore know that $K_{N, f}(x)=0$ if and only if $K_{-N, f}(x)=0$.

REMARKS. (a) $K_{f}(x)$ is a smooth function on $X$.

(b) The Gauss-Bonnet formula tells us, if $X$ is connected, oriented and compact, then $\int_{X} K_{f}=\chi(X)=$ the Euler Characteristic of $X$.

DefinItion 2.2. A point $x \in X$ is called a parabolic point of the immersion $f$ if $K_{N, f}(x)=0$. Corresponding to each immersion $f: X \rightarrow R^{n+1}$ we have the Gauss normal map $G(f): X \rightarrow P_{n}(R)$, which we recall is defined as follows. Let $x \in X$. Let $N_{x}$ be a normal vector to the $n$-plane $T_{1}(f)\left(T_{1}(X)_{x}\right)$ passing through $f(x)$. Let $G(f)(x)$ be the ray through the origin of $R^{n+1}$ determined by $A_{f(x)}\left(N_{x}\right)$.

Proposition 2.3. Let $f \in I\left(X, R^{n-1}\right) . x \in X$ is a parabolic point of $f$ if and only if $x$ is not a regular point of $G(f)$.

Proof. Let $S^{n}$ be the unit $n$-sphere in $R^{n+1}$. Let $\pi: S^{n} \rightarrow P_{n}(R)$ be the antipodal map. It is well known that $\left(\pi: S^{n} \rightarrow P_{n}(R)\right)$ is a twofold covering. Hence if we are interested in studying the singular points (in the usual sense of the word) of $G(f)$, we need only consider the locally defined spherical map which we will now define. 
Let $x \in X$, let $U$ be a small neighborhood of $x$ and assume $f \mid U$ is an embedding. Pick one of the two possible unit normal vectors to $f(U)$ at $f(x)$. Denote this vector by $N_{x}$. We assume that $U$ is small enough so that it is possible to pick, in a smooth manner, unit normals $N_{y}$ to $f(U)$ at $f(y)$ for each $y \in U$, so that $N_{x}$ is the vector chosen above. Then define

$$
\operatorname{Sp}(f)(y)=A_{f(y)}\left(N_{y}\right) \quad \text { for each } y \in U .
$$

It is then clear that $\pi \operatorname{Sp}(f)=G(f) \mid U$. Hence $x$ is a singular point of $\operatorname{Sp}(f)$ if and only if it is a singular point of $G_{N}(f)$.

Let $\left(y_{1}, \ldots, y_{n+1}\right)$ and $\left(\tilde{e}_{1}, \ldots, \tilde{e}_{n+1}\right)$ be the usual coordinates and frames in $R^{n+1}$. Let $U$ be a coordinate neighborhood of $x$ with coordinate functions $\left(x_{1}, \ldots, x_{n}\right)$. Let $f_{k}\left(x_{1}, \ldots, x_{n}\right)=y_{k}\left(f\left(x_{1}, \ldots, x_{n}\right)\right)$. Let us define $C^{\infty}$ functions $\Gamma_{i j}^{k}(x)$ and $h_{i j}(x)$ on $U$, by the formulas

$$
\nabla_{y}\left(\left.\frac{\partial^{2}}{\partial x_{i} \partial x_{j}}\right|_{y}\right)=\left.\sum_{1 \leqq k \leqq n} \Gamma_{i j}^{k}(y) \frac{\partial}{\partial x_{k}}\right|_{y}
$$

and

$$
\nu_{f}\left(\left.\frac{\partial}{\partial x_{i}}\right|_{y},\left.\frac{\partial}{\partial x_{j}}\right|_{y}\right)=h_{i j}(y) N_{y}
$$

for all $y \in U$. Hence we see that

$$
\begin{aligned}
O(f)\left(\left.\frac{\partial^{2}}{\partial x_{i} \partial x_{j}}\right|_{y}\right) & =\left(\lambda \pi_{f}+T_{1}(f) ! \xi\right) w_{2} T_{2}(f) !\left(\left.\frac{\partial^{2}}{\partial x_{i} \partial x_{j}}\right|_{y}\right) \\
& =\lambda v_{f}\left(\left.\frac{\partial}{\partial x_{i}}\right|_{y},\left.\frac{\partial}{\partial x_{j}}\right|_{y}\right)+T_{1}(f) ! \nabla\left(\left.\frac{\partial^{2}}{\partial x_{i} \partial x_{j}}\right|_{y}\right)
\end{aligned}
$$

by standard diagram chasing on Diagram 1.1. Hence

$$
\sum_{k=1}^{n+1} \frac{\partial^{2} f_{k}}{\partial x_{i} \partial x_{j}} \tilde{e}_{k}=\sum_{1 \leqq k \leqq n ; 1 \leqq s \leqq n+1}\left(\Gamma_{i j}^{k} \frac{\partial f_{s}}{\partial x_{k}} \tilde{e}_{s}+h_{i j} N_{s} \tilde{e}_{s}\right)
$$

where $N_{s}$ are $C^{\infty}$ functions on $U$ which are defined by

$$
N_{y}=\sum_{s=1}^{n+1} N_{s}(y) \tilde{e}_{s}(y) \quad \text { for each } y \in U
$$

We will now make an additional abridgement of notation. Let a vector

$$
v=\sum_{i} v_{i} e_{i}(f(x))
$$

at $f(x)$ be considered as the $n+1$ tuple $\left(v_{1}, \ldots, v_{n+1}\right)$. (I.e., as $A_{f(x)}(v)$.) Then the above formula is equivalent to

$$
\frac{\partial^{2} f}{\partial x_{i} \partial x_{j}}=\sum_{k=1}^{n} \Gamma_{i j}^{k} \frac{\partial f}{\partial x_{k}}+h_{i j} N
$$


We know that $\operatorname{Sp}(f)(y)=A_{f(y)} N_{y}$. Hence $\operatorname{Sp}(f)$ is nonsingular if and only if the $n+1$ tuples $\left(\partial N / \partial x_{1}\right) \cdots\left(\partial N / \partial x_{n}\right)$ are linearly independent. But $\left\langle N, \partial f \mid \partial x_{i}\right\rangle=0$. Hence

$$
0=\left(\partial / \partial x_{i}\right)\left\langle N, \partial f \mid \partial x_{j}\right\rangle=\left\langle\partial N / \partial x_{i}, \partial f \mid \partial x_{j}\right\rangle+\left\langle N, \partial^{2} f \mid \partial x_{i} \partial x_{j}\right\rangle
$$

Therefore

$$
-h_{i j}=\left\langle-N, \partial^{2} f \mid \partial x_{i} \partial x_{j}\right\rangle=\left\langle\partial N / \partial x_{i}, \partial f \mid \partial x_{j}\right\rangle .
$$

$\langle N, N\rangle=1$ implies $\partial N /\left.\partial x_{i}\right|_{x} \in T(f) T(X)_{x}$. Hence the vectors $\partial N /\left.\partial x_{i}\right|_{y}$ are tangent to $f(U)$. Thus the vectors $\left(\partial N / \partial x_{i}\right)$ are linearly independent if and only if $\operatorname{det}\left(-h_{i j}\right) \neq 0$. But this is just the matrix of the quadratic form $Q_{N}$ with respect to the basis $\partial / \partial x_{i}$.

Our next discussion will be local in nature. We will construct a model $\left(J^{2}(n) \times O(n+1)\right)$ second order Euclidean singularity $P \subseteq \operatorname{Hom}\left(R^{v(n)}, R^{n+1}\right)$ (see Definition 1.2). Let $P\left(X, R^{n+1}\right) \subseteq \operatorname{Hom}\left(T_{2}(X), T_{1}\left(R^{n+1}\right)\right)$ be the second order Euclidean singularity induced by $P$. $P$ will have the crucial property that $w_{2} T_{2}(f)(x) \in P\left(X, R^{n+1}\right)$ if and only if $x$ is a parabolic point of $f \in I\left(X, R^{n+1}\right)$.

Let $U$ be a coordinate neighborhood of $x \in X$, with coordinate functions $\left(x_{1}, \ldots, x_{n}\right)$. Let $\left(y_{1}, \ldots, y_{n+1}\right)$ and $\left(\tilde{e}_{1}, \ldots, \tilde{e}_{n+1}\right)$ be the usual coordinate functions and vector fields in $R^{n+1}$. Then this gives rise to the usual set of coordinates on Hom $\left(R^{v(n)}, R^{n+1}\right)$. We will view the elements of this vector space as the $(n+1)(v(n))$ tuples of real numbers $\left(a_{i}^{k}, a_{s, t}^{k}\right)(k=1, \ldots, n+1 ; i, s, t=1, \ldots, n ; s \leqq t)$. The $J^{2}(n) \times O(n+1)$ action on this cell is given in the usual way by the chain rule applied to changes of coordinates in $X$ and rigid motions in $R^{n+1}$.

By way of motivating the construction of the desired singular variety we will make the following calculation. Let $f \in I\left(X, R^{n+1}\right)$. Assume $f \mid U$ is an embedding, and assume we can pick $N \in S\left(N_{f}(X)\right)$ such that $\|\lambda N\|_{y}=1$ for each $y \in U$. In this calculation we will identify $R^{n+1}$ with the tangent space at a point. We want to calculate $h_{i j}=\left\langle N, \partial^{2} f \mid \partial x_{i} \partial x_{j}\right\rangle$. We know that $N$ is a unit normal to the $n$-plane $\partial f / \partial x_{1} \wedge \cdots \wedge \partial f / \partial x_{n}$ in $R^{n+1}$ at $f(x)$. Hence

where

$$
\left\langle N, \partial^{2} f \mid \partial x_{i} \partial x_{j}\right\rangle=\frac{\partial f / \partial x_{1} \wedge \cdots \wedge \partial f / \partial x_{n} \wedge \partial^{2} f / \partial x_{i} \partial x_{j}}{\left(\operatorname{Det}\left(g_{i j}\right)\right)^{1 / 2}}
$$

Hence

$$
g_{i j}=\left\langle\partial f\left|\partial x_{i}, \partial f\right| \partial x_{j}\right\rangle
$$

$$
h_{i j}=\operatorname{Det}\left(\begin{array}{ccc}
\frac{\partial^{2} f_{1}}{\partial x_{i} \partial x_{j}} & \cdots & \frac{\partial^{2} f_{n+1}}{\partial x_{i} \partial x_{j}} \\
\frac{\partial f_{1}}{\partial x_{1}} & \cdots & \frac{\partial f_{n+1}}{\partial x_{1}} \\
\cdot & \cdot & \cdot \\
\cdot \\
\frac{\partial f_{1}}{\partial x_{n}} & \cdots & \frac{\partial f_{N=1}}{\partial x_{n}}
\end{array}\right)\left(\operatorname{Det}\left(\sum_{s=1}^{n+1} \frac{\partial f_{s}}{\partial x_{i}} \frac{\partial f_{s}}{\partial x_{j}}\right)\right)^{-1 / 2} \cdot
$$


It is clear that the $h_{i j}$ calculated above motivates the study of the polynomials $\tilde{\phi}_{i j} \in R\left[a_{i}^{k} ; a_{s, t}^{k}\right]$, where

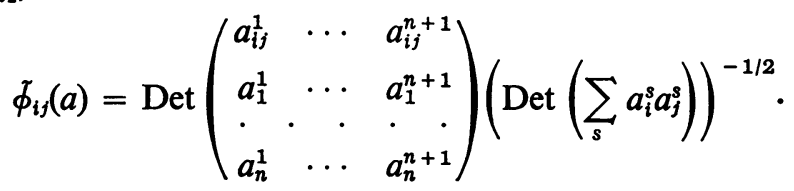

Hence we can restrict our attention to the polynomials

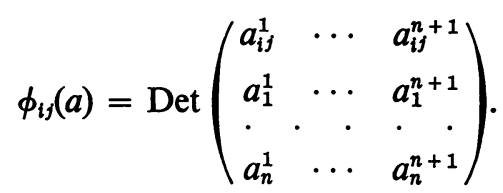

Let an element $b=\left(b_{i}^{j}, b_{i}^{s, t}\right)(i, j, s, t=1, \ldots, n ; s \leqq t)$ represent in the usual way an element of $J^{2}(n)$. Let $b \cdot a$ be the image of $a \in \operatorname{Hom}\left(R^{v(n)}, R^{n+1}\right)$ under the action of $b \in J^{2}(n)$. It is easy to see that

$$
\phi_{i, j}(b \cdot a)=\sum_{s, t=1}^{n} b_{i}^{s} b_{j}^{t} \tilde{\phi}_{s, t}(a) .
$$

We now define the singular variety $P$ by

$$
P=\left\{a \in \operatorname{Hom}\left(R^{v(n)}, R^{n+1}\right) \mid \operatorname{det}\left(\phi_{i j}(a)\right)=0\right\} .
$$

In that $b \in J^{2}(n)$, det $\left(b_{i}^{s}\right) \neq 0$. Hence it is clear from the above formula that $P$ is left invariant by the $J^{2}(n) \times O(n+1)$ action on Hom $\left(R^{\nu(n)}, R^{n+1}\right)$. Hence by our very mode of definition we know that the variety $P$ has the desired properties.

Due to the fact that we are only considering immersions, the only points on the variety $P$ that are of interest are those where the $n+1$ tuples $\left(a_{i}^{1}, \ldots, a_{i}^{n+1}\right)$, $i=1, \ldots, n$ are linearly independent. We will now look for the regular points on this variety under the above restriction. By using normal coordinates we will see that $a \in P$ is a regular point (with the above restriction) if and only if the rank of $\left(\phi_{i j}(a)\right)$ viewed as a matrix is $n-1$.

Let $\Phi_{i j}$ equal the $(i, j)$ th cofactors of the matrix $\phi=\left(\phi_{i j}\right)$. We know that

and

$$
\frac{\partial \phi}{\partial a_{i}^{k}}=\sum_{s, t} \Phi_{s, t} \frac{\partial \phi_{s, t}}{\partial a_{i}^{k}}
$$

$$
\frac{\partial \phi}{\partial a_{i j}^{k}}=\sum_{s, t} \Phi_{s, t} \frac{\partial \phi_{s, t}}{\partial a_{i j}^{k}}
$$

Furthermore we know by hypothesis that the row vectors $\left(a_{i}^{1}, \ldots, a_{i}^{n+1}\right), i=1, \ldots, n$ are linearly independent. Hence there exists some $k^{\prime}$ such that

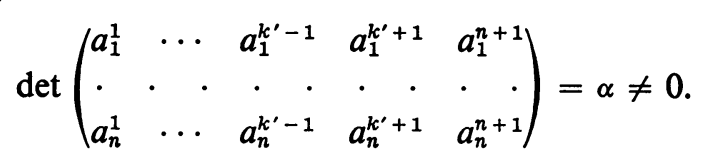

Hence $\partial \phi_{s, t} / \partial a_{i, j}^{k^{\prime}}=\delta_{i, s} \delta_{j, t} \alpha$. Therefore, $\partial \phi / \partial a_{i j}^{k^{\prime}}=\Phi_{i j} \alpha$. This shows our contention. 
We will not worry about how we decompose the variety $P$ into a submanifold collection. We know that this can be done in such a way that the regular points of the variety and the regular points of the submanifold collection coincide (see [5]). We will merely assume that such a decomposition is given.

DEFINITION 2.4. Let $P\left(X, R^{n+1}\right)$ be the Euclidean singularity induced by the model $J^{2}(n) \times O(n+1)$ singularity $P$. Let $f \in I\left(X, R^{n+1}\right)$. We say $f$ is parabolic generic if $O(f)^{\wedge}(X) \pitchfork P\left(X, R^{n+1}\right)$. Theorem 1.3 yields the following result.

THEOREM 2.5. Let $X$ be an n-dimensional smooth manifold. Then the set of parabolic generic immersions in $I\left(X, R^{n+1}\right)$ is dense in $I\left(X, R^{n+1}\right)$.

We can now apply Theorem 1.4. Let $W_{k}(X)$ be the $k$ th Stiefel-Whitney class of $X$. Recall, that $X$ is immersible in $R^{n+1}$ hence $W_{i}(X)=W_{1}(X)^{i}$.

THEOREM 2.6. Let $X$ be a connected n-dimensional smooth manifold. Assume that $X$ is immersible in $R^{n+1}$. Let $f \in I\left(X, R^{n+1}\right)$. Let $S_{p}(f)$ be the set of parabolic points of $f$. Let $i$ be the inclusion of $S_{p}(f)$ in $X$, and let $D_{X}$ be Poincaré duality in $X$. If $f$ is parabolic generic then $S_{p}(f)$ is a manifold collection of dimension $n-1$ if it is not empty. (If the $\operatorname{dim} X$ is even and if $\chi(X) \neq 0$ then the Gauss-Bonnet formula insures us that $S_{p}(f)$ is not empty.) It furthermore carries a fundamental class $\left[S_{p}(f)\right]$, which in addition satisfies the formula

$$
D_{X} i_{*}\left[S_{p}(f)\right]=\varepsilon(n) W_{1}(X),
$$

where $\varepsilon(n)$ is a $Z_{2}$ valued function of the dimension $n$ only.

COROLlARY 2.7. Let $X$ be a connected compact, orientable, n-dimensional manifold. Let $f \in I\left(X, R^{n+1}\right)$ be parabolic generic. Let $i: S_{p}(f) \rightarrow X$ be the inclusion map. Then $S_{p}(f)$ carries a fundamental class $\left[S_{p}(f)\right]$ and $i_{*}\left[S_{p}(f)\right]=0$. (In this case the homology in question can be singular or simplicial theory in that $X$ is compact.)

3. Parabolic points. II. In the light of Proposition 2.3 we could study parabolic points by studying the singularities (in the usual sense) of the smooth vector bundle homomorphism $T_{1}(G(f)): T_{1}(X) \rightarrow T_{1}\left(P_{n}(R)\right)$ covering $G(f): X \rightarrow P_{n}(R)$. Let us denote $P_{n}(R)$ by $P_{n}$ as no confusion will arise in this section.

Let us look at the vector bundle Hom $\left(T_{1}(X), T_{1}\left(P_{n}\right)\right)$. It is a vector bundle with fiber $\operatorname{Hom}\left(R^{n}, R^{n}\right)$ and structural group $G L(n, R) \times G L(n, R)$ which acts on the fiber in the usual way. Let

$$
S_{i}=\left\{\sigma \in \operatorname{Hom}\left(R^{n}, R^{n}\right) \mid \operatorname{rank} \sigma=n-i\right\} .
$$

Then $S_{i}$ is invariant under the action of $G L(n) \times G L(n)$ on Hom $\left(R^{n}, R^{n}\right)$. We see $S=S_{1} \cup \cdots \cup S_{n}$ is a submanifold collection, as well as a real algebraic variety. We can easily compute codim $\left(S_{i}\right)=(i)^{2}$ (see $[2, \S 4]$ ). Therefore we have induced a submanifold collection

$$
S\left(X, P_{n}\right)=S_{1}\left(X, P_{n}\right) \cup \cdots \cup S_{n}\left(X, P_{n}\right)
$$


of Hom $\left(T_{1}(X), T_{1}\left(P_{n}\right)\right)$ where codim $S_{i}\left(X, P_{n}\right)=\operatorname{codim} S_{i}$. Finally we see

$$
\begin{aligned}
P_{i}(f) & =\left\{x \in X\left|T_{1}\left(G_{N}(f)\right)\right| T_{1}(X)_{x} \text { has kernel rank }=i\right\} \\
& =\left\{x \in X \mid T_{1}(G(f))^{\wedge}(x) \in S_{i}\left(X, P_{n}\right)\right\}
\end{aligned}
$$

where

$$
T_{1}(G(f))^{\wedge}: X \rightarrow \operatorname{Hom}\left(T_{1}(X), T_{1}\left(P_{n}\right)\right)
$$

is the smooth cross section over the graph of $G(f)$ defined by

$$
T_{1}(G(f))^{\wedge}(x)=T_{1}(G(f)) \mid T_{1}(X)_{x} .
$$

Definition 3.1. Let $f \in I\left(X, R^{n+1}\right)$. If $T_{1}(G(f))^{\wedge}(X) \pitchfork S_{i}\left(X, P_{n}\right)$ for all $i$ then we say $f$ is strongly parabolic generic.

LEMMA 3.2. Let $f \in I\left(X, R^{n+1}\right)$ and let $x_{0}$ be a parabolic point of $f$. Let $N$ be a normal field such that $N$ is a unit normal vector field in some neighborhood of $x_{0}$.

(a) $\operatorname{rank}\left(S_{N} \mid T_{1}(X)_{x_{0}}\right)=\operatorname{rank}\left(T_{1}\left(G_{N}(f)\right) \mid T_{1}(X)_{x_{0}}\right)$.

(b) If the above rank is $n-1$, then $O(f)^{\wedge}$ is transversal to $P\left(X, R^{n+1}\right)$ at $O(f)^{\wedge}\left(x_{0}\right)$ if and only if $T_{1}(G(f))^{\wedge}$ is transversal to $S_{1}\left(X, P_{n}\right)$ at $T_{1}(G(f))^{\wedge}\left(x_{0}\right)$.

Proof. Part (a) follows immediately from the calculation in Proposition 2.3.

Let $\left(x_{1}, \ldots, x_{n}\right)$ be Riemann normal coordinates centered at $x_{0}$ defined on a coordinate neighborhood $U$ of $x_{0}$. Hence

$$
\left\langle\partial\left|\partial x_{i}\right|_{0}, \partial /\left.\partial x_{j}\right|_{0}\right\rangle_{0}=\delta_{i j}
$$

We can also assume $f\left(x_{0}\right)=0$, and pick an orthonormal frame $e_{1} \cdots e_{n+1}$ of $R^{n+1}$ such that $\left.T_{1}(f)_{0}\left(\partial / \partial x_{i}\right)\right|_{0}=e_{i}$ for $1 \leqq i \leqq n$ and $e_{n+1}$ is the positive unit normal to $f(X)$ at 0 . Let $\Psi_{i}^{k}$ and $\Psi_{s, t}^{k}$ be the coordinates on the fiber

$$
\operatorname{Hom}\left(R^{v(n)}, R^{n+1}\right) \cong \operatorname{Hom}\left(T_{2}(X), T_{1}\left(R^{n+1}\right)\right)_{\left(x_{0}, 0\right)}
$$

chosen with respect to the $x_{i}$ and the $e_{k}$. Let $\pi: \operatorname{Hom}\left(T_{2}(X), T_{1}\left(R^{n+1}\right)\right) \rightarrow X \times R^{n+1}$ be the bundle projection, and let

$$
\theta: \pi^{-1}\left(U \times R^{n+1}\right) \rightarrow \operatorname{Hom}\left(T_{2}(X)_{x_{0}}, T_{1}\left(R^{n+1}\right)_{0}\right)=\operatorname{Hom}\left(R^{v(n)}, R^{n+1}\right)
$$

be the projection onto the fiber. Let

$$
\begin{aligned}
& a_{i}^{k}\left(x_{1}, \ldots, x_{n}\right)=\Psi_{i}^{\kappa} \theta O(f)^{\wedge}\left(x_{1}, \ldots, x_{n}\right)=\left(\partial f_{k} / \partial x_{i}\right), \\
& 1 \leqq i \leqq n, 1 \leqq k \leqq n+1, \\
& a_{x, t}^{k}\left(x_{1}, \ldots, x_{n}\right)=\Psi_{s t}^{\kappa} \theta O(f)^{\wedge}\left(x_{1}, \ldots, x_{n}\right)=\left(\partial^{2} f_{k} / \partial x_{s} \partial x_{t}\right), \\
& 1 \leqq s \leqq t \leqq n, 1 \leqq k \leqq n+1 .
\end{aligned}
$$

We now see that $a_{i}^{k}\left(x_{0}\right)=\delta_{i k}$ and $a_{s, t}^{k}\left(x_{0}\right)=0$ if $k \neq n$ because $\Gamma_{i j}^{k}\left(x_{0}\right)=0 . a_{0}$ $=\theta O(f)^{\wedge}\left(x_{0}\right)$ is a regular point of the variety

$$
P=\left\{a \in \operatorname{Hom}\left(R^{v(n)}, R^{n+1}\right) \mid \phi(a)=0\right\}
$$


by hypothesis. Let $N_{\phi}\left(a_{0}\right)$ be a normal to $P$ at $a_{0}$. Then $\theta O(f)^{\wedge}$ does not meet $P$ transversally at $a_{0}$ if and only if

$$
\left.N_{\phi}\left(a_{0}\right) \cdot T_{1}\left(\theta O(f)^{\wedge}\right)\left(\partial / \partial x_{i}\right)\right|_{x_{0}}=0 \quad 1 \leqq i \leqq n,
$$

where $\cdot$ is the obvious inner product in $R^{\nu(n)(n+1)}=\operatorname{Hom}\left(R^{\nu(n)}, R^{n+1}\right)$. We recall that $\phi=\operatorname{det}\left(\phi_{s t}\right)$ where $\phi_{s t}$ are defined in $\S 2$. Hence by direct calculation we see

$$
\left.\left(\partial \phi_{s t} / \partial a_{i}^{k}\right)\right|_{a_{0}}=(-1)^{n} a_{s t}^{n+1}\left(x_{0}\right) \delta_{i k}
$$

and

$$
\left.\left(\partial \phi_{s t} / \partial a_{i j}^{k}\right)\right|_{a_{0}}=(-1)^{n} \delta_{k, n+1} \delta_{s, i} \delta_{t, j}
$$

Let $\Phi_{i j}$ and $A_{i j}^{n+1}$ be respectively the $(i, j)$ th cofactors of the matrices $\left(\phi_{i j}\left(a_{0}\right)\right)$ and $\left(a_{i j}^{n+1}\left(x_{0}\right)\right)$. We then have $\Phi_{i j}\left(a_{0}\right)=A_{i j}^{n+1}\left(x_{0}\right)$. Therefore

$$
\begin{aligned}
\left.\left(\partial \phi / \partial a_{i}^{k}\right)\right|_{a_{0}} & =\sum_{s, t}(-1)^{n} \delta_{i k} A_{s t}^{n+1}\left(x_{0}\right) a_{s t}^{n+1}\left(x_{0}\right) \\
& =(-1)^{n} \delta_{i k} \sum_{t} \sum_{s} a_{s, t}^{n+1} A_{s, t}^{n+1}=(-1)^{n} \delta_{i k} n \operatorname{det}\left(a_{s, t}^{n+1}\left(x_{0}\right)\right)=0,
\end{aligned}
$$

because $a_{0} \in P$. We also calculate that $\left.\left(\partial \phi / \partial a_{i j}^{k}\right)\right|_{a_{0}}=(-1)^{n} 2 A_{i j}^{n+1}\left(x_{0}\right)$ if $i \neq j$ and $\left.\left(\partial \phi / \partial a_{i i}^{k}\right)\right|_{a_{0}}=(-1)^{n} A_{i i}^{n+1}\left(x_{0}\right)$. Hence $\left.N_{\phi}\left(a_{0}\right) \cdot T_{1}\left(\theta O(f)^{\wedge}\right)\left(\partial / \partial x_{k}\right)\right|_{x_{0}}=0$ if and only if

$$
\left.\sum_{i<j} \frac{\partial a_{i j}^{n+1}}{\partial x_{e}}\right|_{x_{0}}(-1)^{n} 2 A_{i j}^{n+1}\left(x_{0}\right)+\left.(-1)^{n} \sum_{i} \frac{\partial a_{i j}^{n+1}}{\partial x_{e}}\right|_{x_{0}} A_{i i}^{n+1}\left(x_{0}\right)=0 .
$$

Therefore $\left.N_{\phi}\left(a_{0}\right) \cdot T_{1}\left(\theta O(f)^{\wedge}\right)\left(\partial / \partial x_{e}\right)\right|_{x_{0}}=0$ for $1 \leqq e \leqq n$ if and only if

$$
\left.\left(\partial / \partial x_{e}\right)\left(\operatorname{Det}\left(a_{i j}^{n+1}\right)\right)\right|_{x_{0}}=\sum_{i, j}\left(\left.\left(\partial a_{i j}^{n+1} / \partial x_{e}\right)\right|_{x_{0}} A_{i j}^{n+1}\left(x_{0}\right)\right)=0 .
$$

Let $\left(b_{1}, \ldots, b_{n+1}\right)$ be homogeneous coordinates in $P_{n}$ determined by $e_{1}, \ldots, e_{n+1}$ in $R^{n+1}$. We know $G(f)\left(x_{0}\right)=(0,0, \cdot, 0,1)$, therefore let

$$
V=\left\{\left(b_{1}, \ldots, b_{n+1}\right) \mid b_{n+1} \neq 0\right\}
$$

and set $p_{i}=b_{i} / b_{n+1}$. The $p_{i}$ are the usual coordinates on $P_{n}$ and $V$ is well known to be a coordinate neighborhood. By shrinking $U$ if necessary we can also assume that $G(f)(U) \subseteq V$. By linear algebra we can set

$$
b_{i}\left(x_{1} \cdots x_{n}\right)=(-1)^{n+1-i} \operatorname{det}\left(\begin{array}{ccccc}
\frac{\partial f_{1}}{\partial x_{1}} & \cdots & \frac{\partial f_{i}}{\partial x_{1}} & \cdots & \frac{\partial f_{n+1}}{\partial x_{1}} \\
\vdots & & & \vdots \\
\frac{\partial f_{1}}{\partial x_{n}} & \cdots & \frac{\partial f_{i}}{\partial x_{n}} & \cdots & \frac{\partial f_{n+1}}{\partial x_{n}}
\end{array}\right) .
$$


Hence we can set $p_{i}\left(x_{1} \cdots x_{n}\right)=p_{i}\left(G(f)\left(x_{1} \cdots x_{n}\right)\right)=b_{i}\left(x_{1} \cdots x_{n}\right) / b_{n+1}\left(x_{1} \cdots x_{n}\right)$. We know $p_{i}\left(x_{0}\right)=0,1 \leqq i \leqq n$, and $b_{n+1}\left(x_{0}\right)=1$. Direct calculations give us the following formulas:

and

$$
\left.\frac{\partial b_{n+1}}{\partial x_{k}}\right|_{x_{0}}=0,\left.\quad \frac{\partial b_{s}}{\partial x_{k}}\right|_{x_{0}}=\left.(-1) \frac{\partial^{2} f_{n+1}}{\partial x_{k} \partial x_{s}}\right|_{x_{0}} \quad \text { for } 1 \leqq s \leqq n
$$

$$
\left.\frac{\partial^{2} b_{s}}{\partial x_{k} \partial x_{e}}\right|_{x_{0}}=\left.(-1) \frac{\partial^{3} f_{n+1}}{\partial x_{k} \partial x_{e} \partial x_{s}}\right|_{x_{0}} \quad \text { for } 1 \leqq s \leqq n .
$$

Hence we see that

$$
\left.\frac{\partial p_{i}}{\partial x_{j}}\right|_{x_{0}}=\left.(-1) \frac{\partial^{2} f_{n+1}}{\partial x_{i} \partial x_{j}}\right|_{x_{0}} \text { and }\left.\frac{\partial^{2} p_{i}}{\partial x_{j} \partial x_{k}}\right|_{x_{0}}=\left.(-1) \frac{\partial^{3} f_{n+1}}{\partial x_{i} \partial x_{j} \partial x_{k}}\right|_{x_{0}} .
$$

Let $\rho$ : Hom $\left(T_{1}(X), T_{1}\left(P_{n}\right)\right)$ be the bundle projection map and let $p_{0}=G(f)\left(x_{0}\right)$. Let $d_{i j}$ be the coordinates on the fiber $\operatorname{Hom}\left(R^{n}, R^{n}\right)=\operatorname{Hom}\left(T_{1}(X)_{x_{0}}, T_{1}\left(P_{n}\right)_{p_{0}}\right)$ chosen with respect to the $x_{i}$ 's and the $p_{j}$ 's and let $\theta: \rho^{-1}(U \times V) \rightarrow \operatorname{Hom}\left(R^{n}, R^{n}\right)$ be the projection onto the fiber. Let

$$
d_{i j}\left(x_{1}, \ldots, x_{n}\right)=d_{i j}\left(\theta T_{1}(G(f))^{\wedge}\left(x_{1}, \ldots, x_{n}\right)\right)=\left(\partial p_{i} / \partial x_{j}\right), \quad 1 \leqq i, j \leqq n .
$$

Let $d_{0}=\theta\left(T_{1}(G(f))^{\wedge}\left(x_{0}\right)\right)$. We know that $d_{0}$ is a regular point of the variety $S=\left\{\left(d_{i j}\right) \in \operatorname{Hom}\left(R^{n}, R^{n}\right) \mid \operatorname{det}\left(d_{i j}\right)=0\right\}$. Let $N_{d}$ be the normal to the variety $S$ at $d_{0}$. Then $T_{1}(G(f))^{\wedge}(X)$ is not transversal to $S\left(X, P_{n}\right)$ at $T_{1}(G(f))^{\wedge}\left(x_{0}\right)$ if and only if $\left.N_{d} \cdot T_{1}\left(\theta T_{1}(G(f))\right) \wedge\left(\partial / \partial x_{i}\right)\right|_{x_{0}}=0$ for $1 \leqq i \leqq n$. Let $D_{i j}=$ the $(i, j)$ th cofactor of the matrix $\left(d_{i j}\right)$. First of all we have calculated $d_{i j}\left(x_{0}\right)=\left.(-1)\left(\partial^{2} f_{n+1} / \partial x_{i} \partial x_{j}\right)\right|_{x_{0}}$ and $\left.\left(\partial d_{i j} / \partial x_{e}\right)\right|_{x_{0}}=\left.(-1)\left(\partial^{3} f_{n+1} / \partial x_{i} \partial x_{j} \partial x_{e}\right)\right|_{x_{0}}$. Hence the above condition for nontransversality is equivalent to $\left.\sum_{i, j}\left(\partial d_{i j} / \partial x_{e}\right)\right|_{x_{0}} D_{i j}\left(x_{0}\right)=0$ for $1 \leqq e \leqq n$ which is equivalent to the condition that $\left.\left(\partial / \partial x_{e}\right)\left(\operatorname{det}\left(\partial^{2} f_{n+1} / \partial x_{i} \partial x_{j}\right)\right)\right|_{x_{0}}=0,1 \leqq e \leqq n$. This expression is identical to the one for $O(f)^{\wedge}$ at the end of the last paragraph because $a_{i j}^{n+1}\left(x_{1} \cdots x_{n}\right)=\partial^{2} f_{n+1} / \partial x_{i} \partial x_{j}$. This completes the proof of this lemma.

THEOREM 3.3. Let $\operatorname{dim} X=2$. (a) The set of parabolic generic maps is open as well as dense in $I\left(X, R^{3}\right)$.

(b) Furthermore if $f$ is parabolic generic it is strongly parabolic generic.

Proof. We will first prove part (a). We will preserve the notation of the discussion preceding 2.4 . We will again assume that the row vectors $\left(a_{i}^{1}, a_{i}^{2}, a_{i}^{3}\right) i=1,2$ are linearly independent. Let $U$ be a neighborhood of $a_{0}$ in

$$
\operatorname{Hom}\left(R^{v(2)}, R^{3}\right)=\operatorname{Hom}\left(R^{5}, R^{3}\right)
$$

such that the above row vector fields are linearly independent throughout $U$. We know from our preceding discussion that

$$
P_{S} \cap U=\left\{a \in U \mid \Phi_{11}(a)=\Phi_{12}(a)=\Phi_{22}(a)=0\right\},
$$

where $P_{S}=$ the set of singular points of the algebraic variety $P$. Now we know

$$
\Phi_{11}(a)=\phi_{22}(a), \quad \Phi_{12}(a)=-\phi_{12}(a) \quad \text { and } \quad \Phi_{22}(a)=\phi_{11}(a) .
$$


Hence our previous calculation tells us that the covector fields $d \Phi_{11}, d \Phi_{12}$, and $d \Phi_{22}$ are linearly independent throughout $U$. Hence $P_{S} \cap U$ is a regular submanifold of $U$ of codimension 3. Let $f \in I\left(X, R^{3}\right)$ and let $Z$ be a neighborhood of $O(f)^{\wedge}(X)$ in Hom $\left(T_{2}(X), T_{1}\left(R^{3}\right)\right)$ so chosen that $P_{S}\left(X, R^{3}\right) \cap Z$ is a closed submanifold of codimension 3 in $Z$. We can then choose a neighborhood $W_{f}$ of $f$ in $I\left(X, R^{3}\right)$ such that $g \in W_{f} O(f)^{\wedge}(X) \subseteq Z$. Then the first part of 3.3 follows from 1.3. The second part is an immediate corollary of Lemma 3.2.

THEOREM 3.4. Let $X$ be a smooth $n$-dimensional manifold, such that $I\left(X, R^{n+1}\right) \neq \varnothing$. Let $f \in I\left(X, R^{n+1}\right)$ be a strongly parabolic generic immersion. Let $S_{p}(f)$ be the set of parabolic points and let $P_{i}$ be the set of points where $T_{1}(G(f))$ has kernel rank $i$. Let $j: S_{p}(f) \rightarrow X$ be the inclusion, we will also denote by $j$ the map $j \mid P_{i}$. Let

$$
C_{s}^{n+2}=(n+2) ! / s !(n+2-s) !
$$

and let $D_{X}$ be Poincaré duality in $X$. Then $\bar{P}_{i}$ carries a fundamental class $\left[\bar{P}_{i}\right]$ and

$$
D_{X} j_{*}\left[\bar{P}_{i}\right]=\operatorname{det}\left(\begin{array}{ccc}
C_{i}^{n+2} & \cdots & C_{2 i-1}^{n+2} \\
C_{i-1}^{n+2} & \cdots & C_{2 i-2}^{n+2} \\
\vdots & & \vdots \\
C_{1}^{n+2} & \cdots & C_{i}^{n+2}
\end{array}\right) W_{1}(X)^{i^{2}}
$$

Proof. This is an easy application of 4.6 and 4.7 of [2]. In that $X$ is immersible in $R^{n+1}$, we know by Whitney duality that $W_{i}(X)=W_{1}(X)^{i}, \bar{W}_{1}(X)=W_{1}(X)$, and $\bar{W}_{i}(X)=0$ for $i \geqq 2$. Let $\partial \in H^{1}\left(P_{n}(R), Z_{2}\right)$ be a generator. Then $W\left(P_{n}(R)\right)=(1+\partial)^{n+1}$. Let $\xi$ be the canonical line bundle over $P_{n}(R)$, then $G(f)^{-1}(\xi) \cong N_{f}(X)$. Hence $G(f)^{*}(\partial)=\bar{W}_{1}(X)=W_{1}(X)$, and therefore $G(f)^{*}\left(W\left(P_{n}\right)\right)=\left(1+W_{1}(X)\right)^{n+1}$. Hence $G(f)^{*} W\left(P_{n}(R)\right) \cup \bar{W}(X)=\left(1+W_{1}(X)\right)^{n+2}$. The theorem then follows by plugging this into the formulas of 4.6 of [2].

Corollary 3.5. Let $\operatorname{dim} X=2$ and let $X$ be compact and connected. Let $f \in I\left(X, R^{3}\right)$ be parabolic generic. Then $S_{p}(f)$ is a closed submanifold of $X$ of dimension one. (I.e., a set of disjoint circles.) Then $S_{p}(f)$ has a fundamental class $\left[S_{p}(f)\right]$. Let $i: S_{p}(f) \rightarrow X$ be the inclusion map. Then

$$
i_{*}\left[S_{p}(f)\right]=0 .
$$

(The set of circles is homologous to zero.)

COROLlaRY 3.6. Let $X$ be compact, odd dimensional, and unoriented. Then any immersion of $X$ into $R^{n+1}$ has parabolic points.

Proof. The immersions with no parabolic points are strongly parabolic generic. Hence by 3.4 ,

$$
D_{X} j_{*}\left[\bar{P}_{1}\right]=W_{1}(X) \neq 0,
$$

where $\bar{P}_{1}=$ set of parabolic points of $f$, which is clearly impossible. The following nice elementary proof was communicated to me in a letter from W. Pohl. Pohl's 
proof goes as follows: If $f$ has no parabolic points then $G N(f): X \rightarrow P_{n}(R)$ is a covering map. Hence $X$ is either the sphere $S^{n}$ or $P_{n}(R)$, but these are both oriented. Hence we have a contradiction.

4. Umbilic points. I. Let $X$ be a smooth $n$-dimensional manifold. Assume $n \geqq 2$. As in the last section we will be interested in immersions of $X$ in $R^{n+1}$. We will fix the notations and conventions outlined in the beginning of the last section.

Definition 4.1. Let $f \in I\left(X, R^{n+1}\right)$. Let $x \in X$, and let $U$ be a small coordinate neighborhood of $x$. Pick $N \in S\left(N_{f}(X)\right)$ such that $\|N(y)\|=1$ for each $y \in U$. Then the operator of the second fundamental form $S_{N}$ induces a self adjoint linear operator $S_{N}(x): T(X)_{x} \rightarrow T(X)_{x}$. The eigenvalues $k_{i}(x)$ of this operator on $T(X)_{x}$ are called the principal curvaiures of $f$ at $x$. These numbers are uniquely determined up to sign in the sense that if we picked $-N$ instead of $N$ we would merely change the sign of the $k_{i}(x)$.

Definition 4.2. Let $f \in I\left(X, R^{n+1}\right)$. A point $x \in X$ is called an umbilic point of the immersion $f$ if all the principal curvatures of $f$ at $x$ are equal. Hence it is clear that the property of being an umbilic point is independent of the unit normal chosen in a neighborhood of $x$ at $f(x)$, in Definition 4.1. A point $x \in X$ is called a parabolic umbilic point of the immersion $f$ if it is an umbilic point of $f$, and if all the principal curvatures vanish at it.

Proposition 4.3. $x \in X$ is a parabolic umbilic point of an immersion $f$ if and only if it is a totally geodesic point.

Proof. Pick $f \in I\left(X, R^{n+1}\right)$. Let $u, v \in T(X)_{x}$. Let $N \in S\left(N_{f}(X)\right)$ such that $\|N(y)\|=1$ for $y$ in a neighborhood of $x$. Then

$$
0=\left(S_{N}(x) u, v\right)=\left(v_{f}(u, v), N\right) .
$$

Hence $v_{f}(x) \equiv 0$. Therefore 7.4 of [2] induces the following corollary.

Corollary 4.4. The set of $f \in I\left(X, R^{n+1}\right)$ such that $f$ has no parabolic umbilic points is open and dense in $I\left(X, R^{n+1}\right)$.

Definition 4.5. Let $f \in I\left(X, R^{n+1}\right)$. Endow $X$ with the induced Riemann metric. Let $x_{0} \in X$, and let $N \in S\left(N_{f}(X)\right)$, such that $\|N(y)\|=1$ for all $y$ in a small neighborhood of $x_{0}$. Let $E=T_{1}(X)-\{0\}$. Let $t \in E_{x_{0}}$; we define the normal curvature in the direction $N$ of $t$ by $K_{N}(t)=Q_{N}(t, t)_{x_{0}} \mid\langle t, t\rangle_{x_{0}}$, where $Q_{N}$ is the second fundamental form of $f$. We see immediately that $K_{N}(t)=K_{N}(a t)$ for $a \in R-\{0\}$. $K_{N}: E_{x_{0}} \rightarrow R$ is a smooth function.

Proposition 4.6. $x_{0}$ is an umbilic point of $f$ if and only if $K_{N}$ is a constant on $E_{x_{0}}$.

Proof. Say $x_{0}$ is an umbilic point of $f$. Then every vector $t \in E_{x_{0}}$ is an eigenvector of $S_{N}$. Hence if $\lambda$ is the single possible eigenvalue we see that $K_{N}(t)=\lambda$, for all $t \in E_{x_{0}}$. 
Conversely assume $K_{N}(t)=\lambda$ for all $\lambda$. Let $e_{1}, \ldots, e_{n}$ be a set of orthonormal vectors in $T_{1}(X)_{x_{0}}$. Then $K_{N}\left(e_{i}\right)=\lambda=\left\langle S_{N} e_{i}, e_{i}\right\rangle$. Hence $S_{N} e_{i}=\lambda e_{i}$. Therefore $x_{0}$ is an umbilic point of $f$.

Corollary 4.7. Let $f \in I\left(X, R^{n+1}\right)$. Let $x_{0} \in X$, and let $U$ be a coordinate neighborhood of $x_{0}$, so chosen that $f \mid U$ is an embedding. We furthermore assume that we are given a smooth unit normal vector field $N$ on $f(U)$. Let $\left(x_{1}, \ldots, x_{n}\right)$ be the coordinate functions on $U$. Let $_{i j}=\left\langle\partial / \partial x_{i}, \partial / \partial x_{j}\right\rangle_{x_{0}}$ and let $h_{i j}=Q_{N}\left(\partial / \partial x_{i}, \partial / \partial x_{j}\right)_{x_{0}}$, where $\langle$,$\rangle is the induced Riemann structure on X$, and $Q_{N}$ is the second fundamental form of $f$. Then $x_{0}$ is an umbilic point of $f$ if and only if

$$
h_{s, t}(x) g_{i j}(x)=h_{i j}(x) g_{s, t}(x) \quad \text { for all } i, j, s \text {, and } t .
$$

Let $\left(z_{1}, \ldots, z_{n}\right)$ be another set of coordinate functions on $U$. Let $h_{i j}^{z}$ and $g_{i j}^{z}$ be the components of the second fundamental form and the metric tensor with respect to these coordinates respectively. Then we have functions $z_{1}\left(x_{1}, \ldots, x_{n}\right)$, $\ldots, z_{n}\left(x_{1}, \ldots, x_{n}\right)$ defining an automorphism of $U$. It is well known that

$$
h_{i j}^{x}=\sum_{s, t} \frac{\partial z_{s}}{\partial x_{j}} \frac{\partial z_{t}}{\partial x_{i}} h_{s t}^{z}
$$

and

$$
g_{i j}^{x}=\sum_{s, t} \frac{\partial z_{s}}{\partial x_{j}} \frac{\partial z_{t}}{\partial x_{i}} g_{s t}^{z}
$$

Therefore

$$
h_{i j}^{x} g_{p q}^{x}-h_{p q}^{x} g_{i j}^{x}=\sum_{s, t, k, l} \frac{\partial z_{s}}{\partial x_{i}} \frac{\partial z_{t}}{\partial x_{j}} \frac{\partial z_{k}}{\partial x_{p}} \frac{\partial z_{l}}{\partial x_{q}}\left(h_{s t}^{z} g_{k l}^{z}-g_{s t}^{z} h_{k l}^{z}\right)
$$

Hence the relationship $h_{i j} g_{s t}-g_{i j} h_{s t}=0$, for all $i, j, s$, and $t$ is independent of our choice of coordinates.

We will now use the relations of the last proposition in order to construct a model second order Euclidean singularity $v \subseteq \operatorname{Hom}\left(R^{v(n)}, R^{n+1}\right)$. Let

$$
v\left(X, R^{n+1}\right) \subseteq \operatorname{Hom}\left(T_{2}(X), T_{1}\left(R^{n+1}\right)\right)
$$

be the second order Euclidean singularity induced by $v$. $v$ will have the crucial property that $O(f)^{\wedge}(x) \in v\left(X, R^{n+1}\right)$ if and only if $x$ is an umbilic point of $f \in I\left(X, R^{n+1}\right)$.

Let $U$ be a coordinate neighborhood of $x \in X$, with coordinate functions $\left(x_{1}, \ldots, x_{n}\right)$. Let $\left(y_{1}, \ldots, y_{n+1}\right)$ and $\tilde{e}_{1}, \ldots, \tilde{e}_{n+1}$ be the usual coordinate functions and vector fields on $R^{n+1}$. Then this gives rise to the usual set of coordinates on Hom $\left(R^{v(n)}, R^{n+1}\right)$. We will view elements of this vector space as the $(n+1)(\nu(n, 2))$ tuples of real numbers $\left(a_{i}^{k}, a_{s t}^{k}\right)(k=1, \ldots, n+1 ; i, s, t=1, \ldots, n ; s \leqq t)$. The 
$J^{2}(n) \times O(n+1)$ action on this cell is given in the usual way by changes of coordinates in $X$ and rigid motions in $R^{n+1}$. We know from previous calculations that the $g_{i j}$ correspond to $\sum_{s=1}^{n+1} a_{i}^{s} a_{j}^{s}$. Furthermore the $h_{i j}$ correspond to

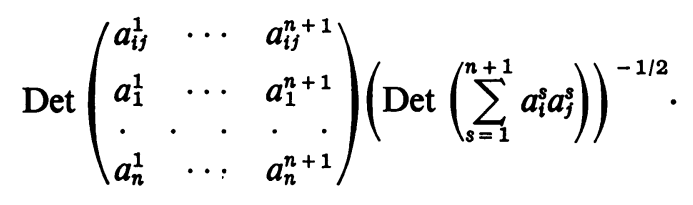

Let

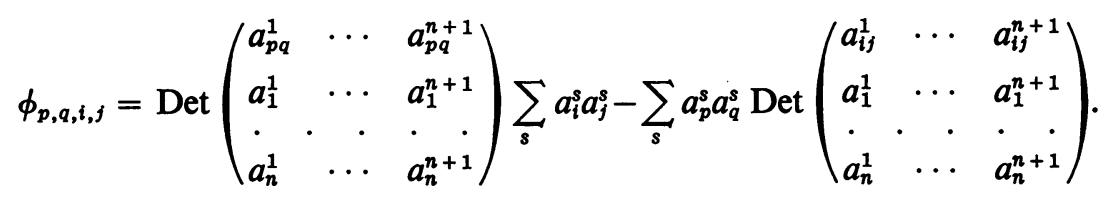

The model singularity that we are interested in is

$$
v=\left\{a \in \operatorname{Hom}\left(R^{v(n)}, R^{n+1}\right) \mid\left(\phi_{p, q, i, j}(a)\right)=0 \text { for all } p, q, i \text {, and } j\right\} .
$$

The calculation at the end of Proposition 4.6 insures that this variety is invariant under the $J^{2}(n) \times O(n+1)$ action on $\operatorname{Hom}\left(R^{v(n)}, R^{n+1}\right)$.

Let $a=\left(a_{i}^{k}, a_{s t}^{k}\right)$ be a point in $\operatorname{Hom}\left(R^{v(n)}, R^{n+1}\right)$ such that the $n+1$-tuples $\left(a_{i}^{1}, \ldots, a_{i}^{n+1}\right), i=1, \ldots, n$, are linearly independent and such that

$$
\operatorname{det}\left(\sum_{s=1}^{n+1} a_{i}^{s} a_{j}^{s}\right) \neq 0 .
$$

Let $V$ be a neighborhood of $a$ so chosen that each point, $a^{\prime} \in V$, has the above two properties. We will now take a look at $V \cap v$. We would like to calculate the codimension of $v \cap V$ in $V$ if the intersection is not void.

We will first show that if $a^{\prime} \in V$ and if $\phi_{11 i j}\left(a^{\prime}\right)=0$ then $a^{\prime} \in v \cap V$. Let

$$
\bar{h}_{i j}\left(a^{\prime}\right)=\operatorname{det}\left(\begin{array}{ccc}
a_{i j}^{\prime} & \cdots & a_{i j}^{\prime n+1} \\
a_{1}^{\prime 1} & \cdots & a_{1}^{\prime n+1} \\
\cdot & \cdot & \cdot \\
a_{n}^{\prime 1} & \cdots & a_{n}^{\prime n+1}
\end{array}\right)
$$

Then

$$
\phi_{11 i j}\left(a^{\prime}\right)=-\bar{h}_{i j}\left(a^{\prime}\right) \sum_{s=1}^{n+1}\left(a_{1}^{\prime s}\right)^{2}+\bar{h}_{11}\left(a^{\prime}\right) \sum_{s=1}^{n+1} a_{i}^{\prime s} a_{j}^{\prime s}
$$

Hence if $\phi_{11 i j}\left(a^{\prime}\right)=0$ for all $i$ and $j$ then

$$
\bar{h}_{i j}\left(a^{\prime}\right)=\bar{h}_{11}\left(a^{\prime}\right) \sum_{s=1}^{n+1} a_{i}^{\prime s} a_{j}^{\prime s} / \sum_{s=1}^{n+1}\left(a_{1}^{\prime s}\right)^{2} .
$$

If $h_{11}\left(a^{\prime}\right)=0$ then $\phi_{p q i j}\left(a^{\prime}\right)=0$ for all $i, j, p$, and $q$. Let us assume that $h_{11}\left(a^{\prime}\right) \neq 0$. 
Then $h_{i j}\left(a^{\prime}\right)=0$ if and only if $\sum_{s=1}^{n+1} a_{i}^{\prime s} a_{j}^{\prime s}=0$. Therefore if $\bar{h}_{i j}\left(a^{\prime}\right)=0$ then $\phi_{p q i j}\left(a^{\prime}\right)=0$ for all $p$ and $q$. Finally if $\sum_{a=1}^{n+1} a_{i}^{s} a_{j}^{s} \neq 0$ then

$$
\bar{h}_{i j}\left(a^{\prime}\right) / \sum_{s} a_{i}^{\prime s} a_{j}^{\prime s}=\bar{h}_{11}\left(a^{\prime}\right) / \sum_{s}\left(a_{1}^{\prime s}\right)^{2}
$$

which implies that $\phi_{p q i}\left(a^{\prime}\right)=0$ for all $p$ and $q$.

In that $\phi_{1111}$ is always zero, the variety $v \cap V$ is given by the vanishing of the $n(n+1) / 2-1$ polynomials $\phi_{11 i j}$. We will now show that the polynomials $\phi_{11 i j}\left(i=j=1\right.$ excluded) are functionally independent at any point $a^{\prime} \in V$.

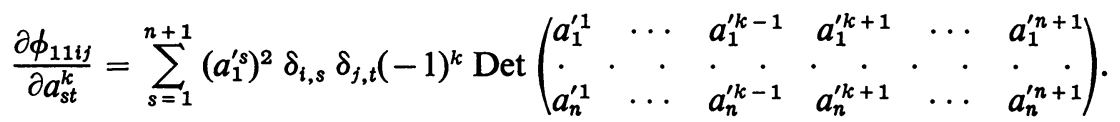

The $\left(a_{1}^{1}, \ldots, a_{i}^{n+1}\right)$ are linearly independent. Hence there exists some $k$ say $k=k^{\prime}$ such that the above determinant is unequal to zero. Hence $\partial \phi_{11 i j} / \partial a_{s, t}^{k^{\prime}}=K \delta_{i s} \delta_{j t}$ where $K$ is a constant unequal to zero, and the cases $s=t=1$, or $i=j=1$ are excluded. Therefore the set $v \cap V$ is a regular submanifold of $V$ of codimension $n(n+1) / 2-1$. Finally, due to the fact that we are only interested in immersions, these are the only points of $\operatorname{Hom}\left(R^{v(n)}, R^{n+1}\right)$ where we are interested in the structure of the variety $v$.

Pick $f \in I\left(X, R^{n+1}\right)$. Let $Z$ be a small tubular neighborhood of $O(f)^{\wedge}(X)$. We can assume that $Z$ is chosen sufficiently small so that $v\left(X, R^{n+1}\right) \cap Z$ is a closed submanifold of codimension $n(n+1) / 2-1$, in $Z$. Then there exists a neighborhood $W$ of $f$ contained in $I\left(X, R^{n+1}\right)$ such that if $g \in W$, then $O(f)^{\wedge}(x) \subseteq Z$. Hence 1.3 gives us the following theorem.

THEOREM 4.8. Let the dimension of $X$ be $\geqq 3$. Then the set of $f \in I\left(X, R^{n+1}\right)$ such that $f$ has no umbilic points is open and dense in $I\left(X, R^{n+1}\right)$.

Before stating our result in the case where $\operatorname{dim} X=2$, we will state a little history about the problem of counting umbilic points. The principal interest in this problem stems from the following conjecture of $\mathrm{C}$. Carathéodory.

C. Carathéodory Conjecture. Let $f: S^{2} \rightarrow R^{3}$ be a convex smooth embedding. Carathéodory conjectured that $f$ has at least two umbilic points. The conjecture in this generality is still outstanding. However, if we restrict ourselves to the category of real analytic maps and manifolds, there have been two proofs to date. The first was given by H. Hamberger (Acta Math. 73, pp. 175-332). A much shorter proof was given by G. Bol (Math. Z. 49, pp. 389-410). Bol's proof had a gap in it that was filled by T. Klotz (Comm. Pure Appl. Math. 12, pp. 273-311). Klotz's paper also includes a complete exposition of Bol's proof.

THEOREM 4.9. Let $X$ be a compact two-dimensional manifold. Then there exists an open dense subset $D_{v} \subseteq I\left(X, R^{n+1}\right)$ of maps with the following properties. If $f \in D_{0}$ then

(a) the set of umbilic points $S_{v}(f)$ of $f$ is a finite set of points and

(b) (the number of umbilic points) $\bmod 2=0$. 
Proof. Let $D_{v}=\left\{f \in I\left(X, R^{n+1}\right) \mid O(f)^{\wedge}(X) \hbar v\left(X, R^{n+1}\right)\right.$. Hence $D_{v}$ is open and dense in $I\left(X, R^{n+1}\right)$ by Proposition 1.3. Let $f \in D_{v}$. By the calculation immediately preceding Theorem 4.8 we know that $S_{v}(f)=\left(O(f)^{\wedge}\right)^{-1}\left(v\left(X, R^{n+1}\right)\right)$ is a closed submanifold of codimension 2 . Therefore we have part (a) of this theorem. Finally Theorem 1.4 tells us, if $f \in D_{v}$ then $S_{v}(f)$ carries a fundamental class $\left[S_{v}(f)\right]$. It furthermore yields that $D_{X} i_{*}\left[S_{U}(f)\right]=$ a polynomial in the Stiefel-Whitney classes. Hence the only possibilities are 0 and $W_{1}(X)^{2}$. An examination of Bol's surface yields our result.

Theorem 5.6 in the next section will also imply this result.

CoROllary 4.10. If $X$ is orientable, if $\chi(X) \neq 0$, and if $f \in D_{v}$, then the number of umbilic points of $f$ is greater than or equal to two.

Proof. By the Poincaré theorem on singularities of vector fields on surfaces $f$ must have one umbilic point. However Theorem 4.9 says that the number of umbilic points is even if $f \in D_{v}$. Hence there are at least two.

In the next section we will improve this result by calculating the index of an isolated umbilic point of an immersion in $D_{v}$.

DeFINITION 4.11. Let us call the maps $f \in D_{0}$ umbilic generic maps.

5. Umbilic points. II. In this section we will deal with the case where we are immersing a 2-dimensional manifold into $R^{3}$. The object is to prove that the index of an isolated umbilic of an umbilic generic map is $\pm \frac{1}{2}$. This section is broken up into three parts. The first part discusses the index of an isolated umbilic and calculates it using isothermal parameters. The second section relates isothermal and geodesic parallel coordinates and the third section proves our theorem. For 5.1 through 5.3 we follow [6], Chapter IV, $\$ 1$ rather closely.

(A) Isothermal coordinates and a definition of the index of an isolated umbilic point. Let $f \in I\left(X, R^{3}\right)$, and endow $X$ with Riemann metric induced by $f$. Let $x_{0} \in X$ and let $(U, \phi)$ be isothermal coordinates centered at $x_{0}$. We will assume that $f \mid U$ is an embedding. Let $(x, y)$ be the coordinate functions on $U$. Hence we know that

$$
\langle\partial \mid \partial x, \partial \partial \partial y\rangle_{(x, y)}=0 \text { and }\langle\partial / \partial x, \partial \partial \partial x\rangle_{(x, y)}=\langle\partial / \partial y, \partial \mid \partial y\rangle_{(x, y)}=\lambda(x, y) .
$$

Let $N(x, y)$ be a smooth vector field which is normal to $f(U)$ at $f(x, y)$, so picked that $\left(T_{1}(f)(\partial / \partial x), T_{1}(f)(\partial / \partial y), N(x, y)\right)$ is oriented in the same way as $R^{3}$. Let $S_{N}: S(T(U)) \rightarrow S(T(U))$ be the operator of the second fundamental form on $U$ and let

$$
M(x, y)=\left\langle S_{N}(\partial / \partial x), \partial / \partial y\right\rangle(x, y), \quad L(x, y)=\left\langle S_{N}(\partial / \partial x), \partial / \partial x\right\rangle(x, y),
$$

and

$$
\tilde{N}(x, y)=\left\langle S_{N}(\partial / \partial y), \partial / \partial y\right\rangle(x, y)
$$


Furthermore let $K(x, y), H(x, y), k_{1}(x, y)$, and $k_{2}(x, y)$ be the Gaussian, mean, and principal curvatures respectively on $U$. Hence

(1) $K(x, y)=k_{1}(x, y) k_{2}(x, y)=\left(L \tilde{N}-M^{2}\right) / \lambda^{2}$,

(2) $H(x, y)=\left(k_{1}(x, y)+k_{2}(x, y)\right) / 2=(L(x, y)+\tilde{N}(x, y)) / 2 \lambda(x, y)$ and

(3) the tangent vectors $(\alpha, \beta)$ to the lines of curvature are given by

$$
-M(\alpha)^{2}+(L-\tilde{N}) \alpha \beta+M \beta^{2}=0 .
$$

Let us introduce complex parameters $z=x+i y$ and $\bar{z}=x-i y$. Then it is easy to see that $\partial / \partial z=\frac{1}{2}(\partial / \partial x-i(\partial / \partial y))$ and $\partial / \partial \bar{z}=\frac{1}{2}(\partial / \partial x+i(\partial / \partial y))$. Let

$$
F(z, \bar{z})=\operatorname{Re} F(z, \bar{z})+\operatorname{Im} F(z, \bar{z}) i=P(z, \bar{z})+i Q(z, \bar{z}) .
$$

Then it is easy to see that

and

$$
2(\partial F / \partial z)=(\partial P / \partial x+\partial Q / \partial y)-i(\partial P / \partial y-\partial Q / \partial x)
$$

Let us define

$$
2(\partial F / \partial \bar{z})=(\partial P / \partial x-\partial Q / \partial y)+i(\partial P / \partial y+\partial Q / \partial x)
$$

$$
\Phi(z, \bar{z})=(L-\tilde{N}) / 2-i M .
$$

Proposition 5.1. (a) $|\Phi(z, \bar{z})|=\left(\lambda(z, \bar{z})\left|k_{1}-k_{2}\right|\right) / 2$.

(b) The tangent vectors $d z / d t$ to the lines of curvature on $U$ are given by

$$
\operatorname{Im}\left(\Phi(z, \bar{z})(d z / d t)^{2}\right)=0 .
$$

$$
\Phi(z, \bar{z})=-2(\partial f / \partial z) \cdot \partial N / \partial z \text {. }
$$

Proof.

$$
\left(\frac{L-\tilde{N}}{2}-i M\right)\left(\frac{L-\tilde{N}}{2}+i M\right)=\left(\frac{L-\tilde{N}}{2}\right)^{2}+M^{2}
$$

and

$$
\left(\frac{k_{1}-k_{2}}{2}\right)^{2}=\left(\frac{k_{1}+k_{2}}{2}\right)^{2}-k_{1} k_{2}=\frac{L+\tilde{N}^{2}}{2 \lambda}-\frac{L \tilde{N}-M^{2}}{\lambda^{2}}=\frac{1}{\lambda^{2}}\left(\left(\frac{L-\tilde{N}}{2}\right)^{2}+M^{2}\right) .
$$

$$
\left(\frac{d z}{d t}\right)^{2}=\left(\frac{d x}{d t}+i \frac{d y}{d t}\right)^{2}=\left(\frac{d x}{d t}\right)^{2}-\left(\frac{d y}{d t}\right)^{2}+2 i \frac{d x}{d t} \frac{d y}{d t}
$$

Therefore,

$$
\begin{aligned}
\Phi(z, \bar{z})\left(\frac{d z}{d t}\right)^{2} & =\left(\frac{L-\tilde{N}}{2}-i M\right)\left(\frac{d z}{d t}\right)^{2} \\
& =\text { real part }+i(L-\tilde{N}) \frac{d x}{d t} \frac{d y}{d t}-M\left(\frac{d x}{d t}\right)^{2}+M\left(\frac{d y}{d t}\right)^{2}
\end{aligned}
$$

(c)

$$
\begin{aligned}
2 \frac{\partial f}{\partial z} \cdot \frac{\partial \tilde{N}}{\partial z} & =\frac{1}{2}\left(\frac{\partial f}{\partial x}-i \frac{\partial f}{\partial y}\right) \cdot\left(\frac{\partial \tilde{N}}{\partial x}-i \frac{\partial \tilde{N}}{\partial y}\right) \\
& =\frac{1}{2}\left(\frac{\partial f}{\partial x} \cdot \frac{\partial \tilde{N}}{\partial x}-\frac{\partial f}{\partial y} \cdot \frac{\partial \tilde{N}}{\partial y}\right)+i\left(-\frac{\partial f}{\partial y} \cdot \frac{\partial \tilde{N}}{\partial x}-\frac{\partial f}{\partial y} \cdot \frac{\partial \tilde{N}}{\partial x}\right) \\
& =-\frac{1}{2}[(L-\tilde{N})+i(-2 M)] \\
& =-\Phi(z, \bar{z}) .
\end{aligned}
$$


If isothermal parameters $(x, y)$ on $U$ are replaced by another set of isothermal parameters $(u, v)$ on $U$ with the same orientation, then if we set $w=u+i v$, the map $z \rightarrow w$ from the $z$ into the $w$ plane is holomorphic. We know that

and that

$$
\Phi(z, \bar{z})=-2(\partial f / \partial z) \cdot \partial \tilde{N} / \partial z
$$

$$
\Phi(w, \bar{w})=-2(\partial f / \partial w) \cdot \partial \tilde{N} / \partial w .
$$

Let $z \rightarrow w(z)$ be the change of coordinates above. Then $\partial f / \partial z=\partial f / \partial w \cdot \partial w / \partial z$. Hence

$$
\Phi(z, \bar{z})=\Psi(w, \bar{w})(\partial w / \partial z)^{2} .
$$

Putting these lemmas together we have shown the following theorem.

THEOREM 5.2. Let $X$ be an oriented, Riemannian two-dimensional manifold. Let $\left(U_{\alpha}\right)$ be an oriented open coordinate cover of $X$, such that each coordinate patch $\left(U_{\alpha}\right)=\left(x_{\alpha}, y_{\alpha}\right)$ is a set of isothermal coordinates. Hence using this Riemann structure $R$ we have defined on $X$ a complete analytic structure $C_{R}$. Let $K^{1,0}$ be the complex line bundle of $C^{\infty}$-differential forms of type $(1,0)$ on $X$ and let $\left(K^{1,0}\right)^{2}=K^{1,0} \otimes K^{1,0}$ as complex line bundles. Let $f \in I\left(X, R^{3}\right)$, and assume that $f$ induces the Riemann structure $R$ on $X$. Then we can define an element $F \in S\left(\left(K^{1,0}\right)^{2}\right)$ with the property that $F(x)=0$ if and only if $x$ is an umbilic point of the immersion $f$.

Proof. On $U_{\alpha}=\left(x_{\alpha}, y_{\alpha}\right)$, let $z_{\alpha}=x_{\alpha}+i y_{\alpha}$, etc. We then define $F$ on $U_{\alpha}$ by

$$
F\left(x_{\alpha}, y_{\alpha}\right)=F\left(z_{\alpha}, \bar{z}_{\alpha}\right)=-2\left(\partial f / \partial z_{\alpha} \cdot \partial \tilde{N} / \partial z_{\alpha}\right)\left(d z_{\alpha}\right)^{2} .
$$

DEFINITION 5.3. Let $U$ be a small coordinate neighborhood of an isolated umbilic point $x_{0}$ of $f \in I\left(X, R^{3}\right)$. Hence $x_{0}$ is an isolated singularity for each of the two families of lines of curvatures. Therefore $x_{0}$ has an index with respect to each of these families. However the fact that the families are mutually perpendicular implies that these two indices are equal. Let $C(s)$ be a simple homotopically trivial closed curve in $U$ around the umbilic $x_{0}$. Let us pick one of the families of lines of curvature. Also assume this family has no other singularity in $U$ and that $x_{0}$ does not lie on $C(s)$. Now for every point $C(s)$ of the curve $C$ let $z(s, t)$ be the line of curvature through $C(s)$. Let $d z(s, t) / d t$ be its tangent vector. We can then define the index $j$ of $x_{0}$ by the formula

$$
j=(1 / 2 \pi) \delta(\arg d z(s, t) / d t),
$$

where $\delta$ is the accumulated change of angle between $d z(s, t) / d t$ and a fixed line as we transverse $C$ once in a counterclockwise direction.

Let us assume we are given isothermal parameters $(x, y)$ on $U$ and that $z=x+i y$, etc. Let $z(t)$ be a line of curvature in $U$. Its tangent vector $d z / d t$ satisfies the equation

$$
\operatorname{Im}\left(\Phi(z(t))(d z / d t)^{2}\right)=0
$$

where $\Phi$ is the function of Proposition 5.1. Hence

$$
\arg \left(\Phi(z(t))(d z / d t)^{2}\right)=m \pi, \quad m=\text { integer }
$$


Hence the argument principle implies

$$
\arg (d z / d t)=m \pi / 2-\arg \Phi / 2 .
$$

Hence the index $j$ of $x_{0}$ satisfies

$$
j=-(1 / 4 \pi)(\delta(\arg \Phi))
$$

Proposition 5.4. Let us assume the hypotheses and discussion of 10.1. Let $x_{0} \in U$ be an isolated umbilic point. Then $\Phi\left(x_{0}\right)=0$. Furthermore if

$$
\Phi:\left(R^{2}, x_{0}\right)=\left(U, x_{0}\right) \rightarrow(C, 0)
$$

is nonsingular at $x_{0}$, then the index $j$ of $x_{0}$ is equal to $\pm \frac{1}{2}$.

Corollary. Let us assume the hypotheses of 5.2. Let $s(X) \subseteq\left(K^{1,0}\right)^{2}$, be the zero section of $\left(K^{1,0}\right)^{2}$. Then if $F(X) \hbar s(X)$ the index of each umbilic point is either $+\frac{1}{2}$ or $-\frac{1}{2}$.

REMARK. In the remainder of this section we will show that if $f$ is umbilic generic then $F(U) \cap s(U)$ where $U$ is some connected oriented neighborhood of an isolated umbilic point of $f$. The reason we cannot use all of $X$ is that it may not be oriented and therefore admits no global complex structure.

(B) Geodesic coordinates. Here we will establish an important local relationship between geodesic parallel and isothermal coordinates.

Let $U$ and $N$ be as in the beginning of part (A). Assume that we are given a second set of coordinates $(u, v)$ on $U$. Let $(u, v)$ be $x_{0}$-centered geodesic parallel coordinates. Hence we can assume that

$$
\begin{gathered}
\langle\partial / \partial u, \partial \mid \partial v\rangle_{(u, v)}=0, \quad\langle\partial|\partial u, \partial| \partial u\rangle_{(u, v)}=1,\langle\partial|\partial v, \partial| \partial v\rangle_{(u, v)}=G(u, v)>0, \\
G(0, v)=1, \text { and } \partial G /\left.\partial u\right|_{(0,0)}=0 .
\end{gathered}
$$

Let

$$
S_{N}: S\left(T_{1}(U)\right) \rightarrow S\left(T_{1}(U)\right)
$$

be the operator of the second fundamental form on $U$. As before we define

$$
L(u, v)=\left\langle S_{N}(\partial / \partial u), \partial \mid \partial v\right\rangle(u, v), \quad M(u, v)=\left\langle S_{N}(\partial / \partial u), \partial / \partial v\right\rangle(u, v)
$$

and

$$
\tilde{N}(u, v)=\left\langle S_{N}(\partial / \partial v), \partial / \partial v\right\rangle(u, v) .
$$

Let us define $\Phi(u, v)=\left(\Phi_{1}(u, v), \Phi_{2}(u, v)\right)$, from $U$ into $R^{2}$ by

$$
\Phi_{1}(u, v)=(L(u, v)-\tilde{N}(u, v)) / 2 \quad \text { and } \quad \Phi_{2}(u, v)=-M(u, v) .
$$

We know $F=0$. Hence

$$
H(u, v)^{2}-K(u, v)=((L(u, v)-\tilde{N}(u, v)) / 2 G(u, v))^{2}+M^{2}(u, v) / G(u, v) .
$$


Hence $\Phi_{1}(0,0)=\Phi_{2}(0,0)=0$ if and only if $x_{0}$ is an umbilic point. Hence in terms of geodesic coordinates $(u, v)$ on $U$ we have constructed a function $\Phi: U \rightarrow R^{2}$ such that $\Phi(u, v)=0$ if and only if $(u, v)$ is an umbilic point. In the last section using isothermal coordinates we constructed a function $\Psi: U \rightarrow C$ such that $\Psi(x, y)=\Psi(z, \bar{z})=0$ if and only if $(x, y)$ is an umbilic point.

Proposition 5.5. Let $\left(x_{0}\right) \in U$ be an isolated umbilic point. Let $\Phi(u, v)$ be the function constructed above with $x_{0}$-centered geodesic parallel coordinates. Let $\Psi(z, \bar{z})$ be the function constructed in part (A) using $x_{0}$-centered isothermal coordinates. Then $\Phi(u, v)$ is regular at $(0,0)=x_{0}$ if and only if $\Psi(x, y)$ is.

Proof. We can assume the $\lambda$ of the isothermal coordinates satisfies $\lambda(0,0)=1$. Let $u(x, y)$ and $v(x, y)$ be smooth functions relating the geodesic and isothermal coordinates centered at $x_{0}$ on $U$. Hence

$$
\left(\begin{array}{ll}
\left.\frac{\partial u}{\partial x}\right|_{0} & \left.\frac{\partial v}{\partial x}\right|_{0} \\
\left.\frac{\partial u}{\partial y}\right|_{0} & \left.\frac{\partial v}{\partial y}\right|_{0}
\end{array}\right)=\left(\begin{array}{ll}
1 & 0 \\
0 & 1
\end{array}\right) \in O(2) .
$$

We also know that

$$
\frac{\partial}{\partial x}=\frac{\partial u}{\partial x} \cdot \frac{\partial}{\partial u}+\frac{\partial v}{\partial x} \cdot \frac{\partial}{\partial v} \text { and } \frac{\partial}{\partial y}=\frac{\partial u}{\partial y} \cdot \frac{\partial}{\partial u}+\frac{\partial v}{\partial y} \cdot \frac{\partial}{\partial v} \cdot
$$

Hence

$$
0=\lambda(x, y)\left[\frac{\partial u}{\partial x} \cdot \frac{\partial u}{\partial y}+\frac{\partial v}{\partial x} \cdot \frac{\partial v}{\partial y}\right]
$$

and

$$
\lambda(x, y)=\left(\frac{\partial u}{\partial x}\right)^{2}+G(u, v)\left(\frac{\partial v}{\partial x}\right)^{2}=\left(\frac{\partial u}{\partial y}\right)^{2}+\left(\frac{\partial v}{\partial y}\right)^{2} G(u, v)
$$

Let $\bar{L}, \bar{N}, \bar{M}$ be the components of the second fundamental form calculated in isothermal coordinates. We therefore have

$$
\begin{gathered}
\bar{L}(x, y)=L(u, v)\left(\frac{\partial u}{\partial x}\right)^{2}+2 M(u, v)\left(\frac{\partial u}{\partial x} \cdot \frac{\partial v}{\partial x}\right)+\tilde{N}(u, v)\left(\frac{\partial v}{\partial x}\right)^{2} \\
\bar{M}(x, y)=L(u, v) \frac{\partial u}{\partial x} \cdot \frac{\partial u}{\partial y}+M(u, v)\left(\frac{\partial v}{\partial x} \cdot \frac{\partial u}{\partial y}+\frac{\partial v}{\partial y} \cdot \frac{\partial u}{\partial x}\right)+\tilde{N}(u, v) \frac{\partial v}{\partial x} \cdot \frac{\partial v}{\partial y}
\end{gathered}
$$

and

$$
\bar{N}(x, y)=L(u, v)\left(\frac{\partial u}{\partial y}\right)^{2}+2 M(u, v)\left(\frac{\partial u}{\partial y} \cdot \frac{\partial v}{\partial y}\right)+\tilde{N}(u, v)\left(\frac{\partial v}{\partial y}\right)^{2}
$$


Now

$$
\begin{aligned}
& \left.\frac{\partial \bar{L}}{\partial x}\right|_{0}=\left.\frac{\partial L}{\partial u}\right|_{0}+\left.2 L(0) \frac{\partial^{2} u}{\partial x^{2}}\right|_{0},\left.\quad \frac{\partial \bar{L}}{\partial y}\right|_{0}=\left.\frac{\partial L}{\partial v}\right|_{0}+\left.2 L(0) \frac{\partial^{2} u}{\partial x \partial y}\right|_{0}, \\
& \left.\frac{\partial \bar{M}}{\partial x}\right|_{0}=\left.L(0) \frac{\partial^{2} u}{\partial x \partial y}\right|_{0}+\left.\tilde{N}(0) \frac{\partial^{2} v}{\partial x^{2}}\right|_{0}+\left.\frac{\partial M}{\partial u}\right|_{0} \\
& \frac{\partial \bar{M}}{\partial y}=\left.L(0) \frac{\partial^{2} u}{\partial y^{2}}\right|_{0}+\left.\frac{\partial M}{\partial v}\right|_{0}+\left.\tilde{N}(0) \frac{\partial^{2} v}{\partial x \partial y}\right|_{0} \\
& \left.\frac{\partial \bar{N}}{\partial x}\right|_{0}=\left.\frac{\partial \tilde{N}}{\partial u}\right|_{0}+\left.2 \tilde{N}(0) \frac{\partial^{2} v}{\partial x \partial y}\right|_{0}, \quad \text { and }\left.\frac{\partial \bar{N}}{\partial y}\right|_{0}=\left.\frac{\partial \tilde{N}}{\partial v}\right|_{0}+\left.2 \tilde{N}(0) \frac{\partial^{2} v}{\partial y^{2}}\right|_{0}
\end{aligned}
$$

Therefore

$$
\left.\frac{1}{2}\left(\frac{\partial \bar{L}}{\partial x}-\frac{\partial \bar{N}}{\partial x}\right)\right|_{0}=\frac{1}{2}\left(\left.\frac{\partial L}{\partial u}\right|_{0}-\left.\frac{\partial \tilde{N}}{\partial u}\right|_{0}\right)+\left.L(0) \frac{\partial^{2} u}{\partial x^{2}}\right|_{0}-\left.\tilde{N}(0) \frac{\partial^{2} v}{\partial x \partial y}\right|_{0}
$$

and

$$
\left.\frac{1}{2}\left(\frac{\partial \bar{L}}{\partial y}-\frac{\partial \bar{N}}{\partial y}\right)\right|_{0}=\left.\frac{1}{2}\left(\frac{\partial L}{\partial v}-\frac{\partial \tilde{N}}{\partial v}\right)\right|_{0}+\left.L(0) \frac{\partial^{2} u}{\partial x \partial y}\right|_{0}-\left.\tilde{N}(0) \frac{\partial^{2} v}{\partial y^{2}}\right|_{0}
$$

We know $\partial u / \partial x \cdot \partial u / \partial y+\partial v / \partial x \cdot \partial v / \partial y=0$, hence

$$
\frac{\partial^{2} u}{\partial x \partial y} \cdot \frac{\partial u}{\partial y}+\frac{\partial u}{\partial x} \cdot \frac{\partial^{2} u}{\partial y^{2}}+\frac{\partial^{2} v}{\partial x \partial y} \cdot \frac{\partial v}{\partial y}+\frac{\partial v}{\partial x} \cdot \frac{\partial^{2} v}{\partial y^{2}}=0
$$

and

$$
\frac{\partial^{2} u}{\partial x^{2}} \cdot \frac{\partial u}{\partial y}+\frac{\partial u}{\partial x} \cdot \frac{\partial^{2} u}{\partial x \partial y}+\frac{\partial^{2} v}{\partial x^{2}} \cdot \frac{\partial v}{\partial y}+\frac{\partial v}{\partial x} \cdot \frac{\partial^{2} y}{\partial x \partial y}=0
$$

Hence at $(0,0)$ we have

$$
\left.\frac{\partial^{2} u}{\partial y^{2}}\right|_{0}+\left.\frac{\partial^{2} v}{\partial x \partial y}\right|_{0}=0 \text { and }\left.\frac{\partial^{2} u}{\partial x \partial y}\right|_{0}+\left.\frac{\partial^{2} v}{\partial x^{2}}\right|_{0}=0
$$

Similarly we know $(\partial u / \partial x)^{2}-(\partial u / \partial y)^{2}=G(u, v)\left((\partial v / \partial y)^{2}-(\partial v / \partial x)^{2}\right)$. Therefore

$$
2 \frac{\partial u}{\partial x} \cdot \frac{\partial^{2} u}{\partial x^{2}}-2 \frac{\partial u}{\partial y} \cdot \frac{\partial^{2} u}{\partial y \partial x}=\frac{\partial G}{\partial x} \cdot\left(\left(\frac{\partial v}{\partial y}\right)^{2}-\left(\frac{\partial v}{\partial x}\right)^{2}\right)+G(u, v) \frac{\partial}{\partial x} \cdot\left(\left(\frac{\partial v}{\partial y}\right)^{2}-\left(\frac{\partial v}{\partial x}\right)^{2}\right)
$$

and

$$
2 \frac{\partial u}{\partial x} \cdot \frac{\partial^{2} u}{\partial x \partial y}-2 \frac{\partial u}{\partial y} \cdot \frac{\partial^{2} u}{\partial y^{2}}=\frac{\partial G}{\partial y} \cdot\left(\left(\frac{\partial v}{\partial y}\right)^{2}-\left(\frac{\partial v}{\partial x}\right)^{2}\right)+G(u, v) \frac{\partial}{\partial y} \cdot\left(\left(\frac{\partial v}{\partial y}\right)^{2}-\left(\frac{\partial v}{\partial x}\right)^{2}\right) .
$$

Now we have

$$
\left.\frac{\partial^{2} u}{\partial x^{2}}\right|_{0}=\left.\frac{\partial^{2} v}{\partial x \partial y}\right|_{0} \text { and }\left.\frac{\partial^{2} u}{\partial x \partial y}\right|_{0}=\left.\frac{\partial^{2} v}{\partial y^{2}}\right|_{0}
$$


Hence

$$
\begin{gathered}
\frac{1}{2}\left(\left.\frac{\partial \bar{L}}{\partial x}\right|_{0}-\left.\frac{\partial \bar{N}}{\partial x}\right|_{0}\right)= \\
\frac{1}{2}\left(\left.\frac{\partial L}{\partial u}\right|_{0}-\left.\frac{\partial \tilde{N}}{\partial u}\right|_{0}\right), \quad \frac{1}{2}\left(\left.\frac{\partial \bar{L}}{\partial y}\right|_{0}-\left.\frac{\partial \bar{N}}{\partial y}\right|_{0}\right)=\frac{1}{2}\left(\left.\frac{\partial L}{\partial v}\right|_{0}-\left.\frac{\partial \tilde{N}}{\partial v}\right|_{0}\right), \\
\left.\frac{\partial \bar{M}}{\partial x}\right|_{0}=\left.\frac{\partial M}{\partial u}\right|_{0} \text { and }\left.\frac{\partial \bar{M}}{\partial y}\right|_{0}=\left.\frac{\partial M}{\partial u}\right|_{0} .
\end{gathered}
$$

Therefore det (Jacobian $\Psi(0))=\operatorname{det}($ Jacobian $\Phi(0))$.

(C) Proof of theorem. Let $f \in I\left(X, R^{3}\right)$. Let $x_{0}$ be an isolated umbilic point of $f$. Let $U$ be an $x_{0}$-centered geodesic parallel coordinate neighborhood, and let $(u, v)$ be the coordinate functions. Let $\left(y_{1}, y_{2}, y_{3}\right)$ and $\left(\tilde{e}_{1}, \tilde{e}_{2}, \tilde{e}_{3}\right)$ be the usual coordinates and frames in $R^{3}$. Assume that $f\left(x_{0}\right)$ is the origin of $R^{3}$,

$$
\left.T_{1}(f)_{(0,0)} \frac{\partial}{\partial u}\right|_{0}=\tilde{e}_{1}(0),\left.\quad T_{1}(f) \frac{\partial}{\partial v}\right|_{0}=\tilde{e}_{2}(0),
$$

and that we have chosen a normal vector field $N$ on $f(U)$ so that $N(0)=\tilde{e}_{3}(0)$. Let $O(f): T_{2}(X) \rightarrow T_{1}\left(R^{3}\right)$ be the osculating map. We know that

$$
O(f)\left(\frac{\partial}{\partial u}\right)=\sum_{i=1}^{3} \frac{\partial f_{i}}{\partial u} \tilde{e}_{i}(f(u, v)) \text { and } O(f)\left(\frac{\partial^{2}}{\partial u^{2}}\right)=\sum_{i=1}^{3} \frac{\partial^{2} f_{i}}{\partial u^{2}} \tilde{e}_{i}(f(u, v)) \text {, etc. }
$$

Let $\pi:$ Hom $\left(T_{2}(X), T_{1}\left(R^{3}\right)\right) \rightarrow X \times R^{3}$ be the bundle projection. Let

$$
\theta: \pi^{-1}\left(U \times R^{3}\right) \rightarrow \operatorname{Hom}\left(T_{2}(X), T_{1}\left(R^{3}\right)\right)_{\left(x_{0}, 0\right)}
$$

be the projection onto the fiber. Let $a_{i}^{k}$, and $a_{s, t}^{k}$ be the coordinates on the fiber induced by the $\partial / \partial u, \partial^{2} / \partial u^{2}, \ldots$, and $\partial / \partial y_{i}=\tilde{e}_{i}$. Look at the map $\gamma=\theta O(f)^{\wedge}: U \rightarrow$ Fiber. Let

$$
\begin{aligned}
& a_{1}^{1}(u, v)=a_{1}^{1} \gamma(u, v)=\frac{\partial f_{1}}{\partial u}, \quad a_{1}^{2}(u, v)=a_{1}^{2} \gamma(u, v)=\frac{\partial f_{2}}{\partial u}, \\
& a_{1}^{3}(u, v)=a_{1}^{3} \gamma(u, v)=\frac{\partial f_{3}}{\partial u}, \quad a_{2}^{1}(u, v)=\frac{\partial f_{1}}{\partial v}, \quad a_{2}^{2}(u, v)=\frac{\partial f_{2}}{\partial v}, \\
& a_{2}^{3}(u, v)=\frac{\partial f_{3}}{\partial v}, \quad a_{11}^{1}(u, v)=\frac{\partial^{2} f_{1}}{\partial u^{2}}, \quad a_{11}^{2}(u, v)=\frac{\partial^{2} f_{2}}{\partial u^{2}}, \\
& a_{11}^{3}(u, v)=\frac{\partial^{2} f_{3}}{\partial u^{2}}, \ldots, a_{22}^{3}(u, v)=\frac{\partial^{2} f_{3}}{\partial v^{2}} .
\end{aligned}
$$

In the last section we defined an algebraic variety $v \subseteq$ Fiber $=\operatorname{Hom}\left(R^{5}, R^{3}\right)$ as follows. $v=\left\{a \in\right.$ Fiber $\left.\mid \phi_{11}{ }_{12}(a)=\phi_{1122}(a)=0\right\}$ where

$$
\phi_{1112}=\operatorname{Det}\left(\begin{array}{lll}
a_{11}^{1} & a_{11}^{2} & a_{11}^{3} \\
a_{1}^{1} & a_{1}^{2} & a_{1}^{3} \\
a_{2}^{1} & a_{2}^{2} & a_{2}^{3}
\end{array}\right) \sum_{i=1}^{3} a_{1}^{i} a_{2}^{i}-\sum_{i=1}^{3}\left(a_{1}^{i}\right)^{2} \operatorname{Det}\left(\begin{array}{lll}
a_{12}^{1} & a_{12}^{2} & a_{12}^{3} \\
a_{1}^{1} & a_{1}^{2} & a_{1}^{3} \\
a_{2}^{1} & a_{2}^{2} & a_{2}^{3}
\end{array}\right)
$$

and

$$
\phi_{1122}=\operatorname{Det}\left(\begin{array}{lll}
a_{11}^{1} & a_{11}^{2} & a_{11}^{3} \\
a_{1}^{1} & a_{1}^{2} & a_{1}^{3} \\
a_{2}^{1} & a_{2}^{2} & a_{2}^{3}
\end{array}\right) \sum_{i=1}^{3}\left(a_{2}^{i}\right)^{2}-\sum_{i=1}^{3}\left(a_{1}^{i}\right)^{2} \operatorname{Det}\left(\begin{array}{ccc}
a_{22}^{1} & a_{22}^{2} & a_{22}^{3} \\
a_{1}^{1} & a_{1}^{2} & a_{1}^{3} \\
a_{2}^{1} & a_{2}^{2} & a_{2}^{3}
\end{array}\right) .
$$


Let $a_{0}=\gamma(0,0) . \quad a_{0} \in U$ because $x_{0}$ is an umbilic point. Furthermore by our choice of coordinates we have

$$
\begin{gathered}
a_{1}^{1}\left(a_{0}\right)=a_{2}^{2}\left(a_{0}\right)=1, \\
0=a_{2}^{1}\left(a_{0}\right)=a_{1}^{2}\left(a_{0}\right)=a_{1}^{3}\left(a_{0}\right)=a_{2}^{3}\left(a_{0}\right)=a_{i j}^{1}\left(a_{0}\right)=a_{i j}^{2}\left(a_{0}\right)=a_{12}^{3}\left(a_{0}\right)=0,
\end{gathered}
$$

and $a_{11}^{3}\left(a_{0}\right)=a_{22}^{3}\left(a_{0}\right)$. Let $\phi_{112}=\alpha$ and $\phi_{1122}=\beta$. Hence

$$
\begin{gathered}
\left.\frac{\partial \alpha}{\partial a_{11}^{i}}\right|_{0}=\left.\frac{\partial \alpha}{\partial a_{22}^{i}}\right|_{0}=0 \quad \text { for } i=1,2,3,\left.\quad \frac{\partial \alpha}{\partial a_{12}^{1}}\right|_{0}=\left.\frac{\partial \alpha}{\partial a_{12}^{2}}\right|_{0}=0,\left.\quad \frac{\partial \alpha}{\partial a_{12}^{3}}\right|_{0}=-1, \\
\left.\frac{\partial \alpha}{\partial a_{1}^{1}}\right|_{0}=0,\left.\quad \frac{\partial \alpha}{\partial a_{1}^{2}}\right|_{0}=a_{1}^{3}\left(a_{0}\right),\left.\quad \frac{\partial \alpha}{\partial a_{1}^{3}}\right|_{0}=\left.\frac{\partial \alpha}{\partial a_{2}^{1}}\right|_{0}=\left.\frac{\partial \alpha}{\partial a_{2}^{2}}\right|_{0}=\left.\frac{\partial \alpha}{\partial a_{2}^{3}}\right|_{0}=0, \\
\left.\frac{\partial \beta}{\partial a_{12}^{i}}\right|_{0}=0 \quad i=1,2,3,\left.\quad \frac{\partial \beta}{\partial a_{11}^{1}}\right|_{0}=\left.\frac{\partial \beta}{\partial a_{11}^{2}}\right|_{0}=0,\left.\quad \frac{\partial \beta}{\partial a_{11}^{3}}\right|_{0}=1, \\
\left.\frac{\partial \beta}{\partial a_{22}^{1}}\right|_{0}=\left.\frac{\partial \beta}{\partial a_{22}^{2}}\right|_{0}=0,\left.\quad \frac{\partial \beta}{\partial a_{22}^{3}}\right|_{0}=-1,\left.\quad \frac{\partial \beta}{\partial a^{1}}\right|_{0}=-3 a_{22}^{3}+a_{11}^{3}, \\
\left.\frac{\partial \beta}{\partial a_{2}^{1}}\right|_{0}=\left.\frac{\partial \beta}{\partial a_{1}^{2}}\right|_{0}=\left.\frac{\partial \beta}{\partial a_{1}^{3}}\right|_{0}=\left.\frac{\partial \beta}{\partial a_{2}^{3}}\right|_{0}=0, \quad \text { and }\left.\quad \frac{\partial \beta}{\partial a_{2}^{2}}\right|_{0}=3 a_{11}^{3}-a_{22}^{3} .
\end{gathered}
$$

Therefore the covectors $d \alpha(0)=-d a_{12}^{3}+a_{11}^{3} d a_{1}^{2}+a_{11}^{3} d a_{2}^{1}$ and

$$
d \beta(0)=d a_{11}^{3}-d a_{22}^{3}+\left(a_{11}^{3}-3 a_{22}^{3}\right) d a_{1}^{1}+\left(3 a_{11}^{3}-a_{22}^{3}\right) d a_{2}^{2} .
$$

Let $\mu=\left.T_{1}(\gamma)(\partial / \partial u)\right|_{0}$ and let $\nu=\left.T_{1}(\gamma)(\partial / \partial v)\right|_{0}$. It is an easy matter to see that $(d \alpha \wedge d \beta)(\mu \wedge \nu) \neq 0$ if and only if $\gamma(U)$ meets $v$ transversally at $a_{0}$. Now

$$
(d \alpha \wedge d \beta)(\mu \wedge \nu)=d \alpha(\mu) d \beta(\nu)-d \alpha(\nu) d \beta(\mu) .
$$

We see that

and

$$
\mu=\left.a_{11}^{3}(0) \frac{\partial}{\partial a_{1}^{3}}\right|_{0}+\left.\sum_{k, s, t} \frac{\partial a_{s, t}^{k}}{\partial u} \cdot \frac{\partial}{\partial a_{s, t}^{k}}\right|_{a_{0}}
$$

Hence

$$
\nu=\left.a_{22}^{3}(0) \frac{\partial}{\partial a_{2}^{3}}\right|_{0}+\left.\sum_{k, s, t} \frac{\partial a_{s, t}^{k}}{\partial v} \cdot \frac{\partial}{\partial a_{s, t}^{k}}\right|_{a_{0}} .
$$

and

Hence

$$
d \alpha(\mu)=-\left.\frac{\partial a_{12}^{3}}{\partial u}\right|_{0}, \quad d \alpha(\nu)=-\left.\frac{\partial a_{12}^{3}}{\partial v}\right|_{0}, \quad d \beta(\mu)=\left.\frac{\partial a_{11}^{3}}{\partial u}\right|_{0}-\left.\frac{\partial a_{22}^{3}}{\partial u}\right|_{0}
$$

$$
\begin{aligned}
(d \alpha) \wedge(d \beta)(\mu \wedge v)= & \left(\left.\frac{\partial a_{11}^{3}}{\partial u}\right|_{0}-\left.\frac{\partial a_{22}^{3}}{\partial u}\right|_{0}\right)\left(\left.\frac{\partial a_{12}^{3}}{\partial v}\right|_{0}\right)-\left(\left.\frac{\partial a_{11}^{3}}{\partial v}\right|_{0}-\left.\frac{\partial a_{22}^{3}}{\partial v}\right|_{0}\right)\left(\left.\frac{\partial a_{12}^{3}}{\partial u}\right|_{0}\right) \\
= & \left(\left.\frac{\partial}{\partial u}\left(\frac{\partial^{2} f_{3}}{\partial u^{2}}-\frac{\partial^{2} f_{3}}{\partial v^{2}}\right)\right|_{0}\right)\left(\left.\frac{\partial}{\partial v}\left(\frac{\partial^{2} f_{3}}{\partial u \partial v}\right)\right|_{0}\right) \\
& -\left(\left.\frac{\partial}{\partial u}\left(\frac{\partial^{2} f_{3}}{\partial u \partial v}\right)\right|_{0}\right)\left(\left.\frac{\partial}{\partial v}\left(\frac{\partial^{2} f_{3}}{\partial u^{2}}-\frac{\partial^{2} f_{3}}{\partial v^{2}}\right)\right|_{0}\right) .
\end{aligned}
$$


By using the formulas for $L, M$, and $\tilde{N}$ derived in $\$ 2$ immediately after 2.3 , we see that

$$
\begin{gathered}
\left.\frac{\partial L}{\partial u}\right|_{0}=\left.\frac{\partial^{3} f_{3}}{\partial u^{3}}\right|_{0}, \quad \frac{\partial L}{\partial v}=\left.\frac{\partial^{3} f_{3}}{\partial u^{2} \partial v}\right|_{0},\left.\quad \frac{\partial M}{\partial u}\right|_{0}=\left.\left.\frac{\partial^{3} f_{3}}{\partial u^{2} \partial v}\right|_{0} \quad \frac{\partial M}{\partial v}\right|_{0}=\left.\frac{\partial^{3} f_{3}}{\partial u \partial v^{2}}\right|_{0} \\
\left.\frac{\partial \tilde{N}}{\partial u}\right|_{0}=\left.\frac{\partial^{3} f_{3}}{\partial v^{2} \partial u}\right|_{0} \text { and }\left.\frac{\partial \tilde{N}}{\partial v}\right|_{0}=\left.\frac{\partial^{3} f_{3}}{\partial v^{3}}\right|_{0}
\end{gathered}
$$

Hence

$$
\begin{aligned}
-d \alpha \wedge d \beta(\mu \wedge \nu) & =\left(\left.\frac{\partial L}{\partial u}\right|_{0}-\left.\frac{\partial \tilde{N}}{\partial u}\right|_{0}\right)\left(-\left.\frac{\partial M}{\partial v}\right|_{0}\right)-\left(\left.\frac{\partial M}{\partial u}\right|_{0}\right)\left(\left.\frac{\partial L}{\partial v}\right|_{0}-\left.\frac{\partial \tilde{N}}{\partial v}\right|_{0}\right) \\
& =\frac{1}{2} \operatorname{det}(\text { Jacobian } \Phi(0)),
\end{aligned}
$$

where $\Phi: U \rightarrow R^{2}$ is the map defined in the beginning of part (B) of $\S 5$. Therefore we have shown the following theorem.

THEOREM 5.6. Let $f \in I\left(X, R^{3}\right)$. Let us assume that $x_{0}$ is an isolated umbilic point of $f$. Let $v\left(X, R^{3}\right) \subseteq \operatorname{Hom}\left(T_{2}(X), T_{1}\left(R^{3}\right)\right)$ be the second order Euclidean singularity defined by $v$. (See $\S 4$.) If $O(f)^{\wedge}(X)$ meets $v\left(X, R^{3}\right)$ transversally at $O(f)^{\wedge}\left(x_{0}\right)$ then the index of $x_{0}$ is $\pm \frac{1}{2}$.

Corollary 5.7. Let $f \in I\left(X, R^{3}\right)$. Assume that $f$ is umbilic generic. Then the number of umbilic points $U(f)$ of $f$ satisfies

$$
U(f) \geqq 2|\chi(X)|, \quad \text { where } \chi(X)
$$

is the Euler characteristic of $X$.

REMARK. Corollary 5.7 agrees with Theorem 5.2 because the Chern class $C\left(\left(K^{1,0}\right)^{2}\right)([X])=-2 \chi(X)$, where $([X])$ means evaluating the 2-dimensional integral class on the fundamental 2-cycle $[X]$, of $X$.

\section{BIBLIOGRAPHY}

1. E. A. Feldman, Geometry of immersions. I, Trans. Amer. Math. Soc. 120 (1965), 185-224.

2. - Geometry of immersions. II, Trans. Amer. Math. Soc. 125 (1966), 181-215.

3. —_, Geometry of immersions. II, Bull. Amer. Math. Soc. 70 (1964), 600-607.

4. W. F. Pohl, Differential geometry of higher order, Topology 1 (1962), 169-211.

5. H. Whitney, Elementary structure of real algebraic varieties, Ann. of Math. (2) 66 (1957), 545-556.

6. H. Hopf, Lectures on differential geometry in the large, Mimeographed notes, Stanford Univ., Stanford, Calif., 1956.

7. D. J. Struik, Differential geometry, Addison-Wesley, Reading, Mass., 1950.

Princeton University,

Princeton, NeW Jersey 